\title{
Closed-loop control of boundary layer streaks induced by free-stream turbulence
}

\author{
George Papadakis, ${ }^{1,}{ }^{*}$ Liang Lu, ${ }^{1}$ and Pierre Ricco ${ }^{2}$ \\ ${ }^{1}$ Department of Aeronautics, Imperial College London, London SW7 2AZ, United Kingdom \\ ${ }^{2}$ Department of Mechanical Engineering, University of Sheffield, Sheffield S1 3JD, United Kingdom
}

(Received 12 January 2016; published 26 August 2016)

The central aim of the paper is to carry out a theoretical and numerical study of active wall transpiration control of streaks generated within an incompressible boundary layer by free-stream turbulence. The disturbance flow model is based on the linearized unsteady boundary-region (LUBR) equations, studied by Leib, Wundrow, and Goldstein [J. Fluid Mech. 380, 169 (1999)], which are the rigorous asymptotic limit of the NavierStokes equations for low-frequency and long-streamwise wavelength. The mathematical formulation of the problem directly incorporates the random forcing into the equations in a consistent way. Due to linearity, this forcing is factored out and appears as a multiplicative factor. It is shown that the cost function (integral of kinetic energy in the domain) is properly defined as the expectation of a random quadratic function only after integration in wave number space. This operation naturally introduces the free-stream turbulence spectral tensor into the cost function. The controller gains for each wave number are independent of the spectral tensor and, in that sense, universal. Asymptotic matching of the LUBR equations with the free-stream conditions results in an additional forcing term in the state-space system whose presence necessitates the reformulation of the control problem and the rederivation of its solution. It is proved that the solution can be obtained analytically using an extension of the sweep method used in control theory to obtain the standard Riccati equation. The control signal consists of two components, a feedback part and a feed-forward part (that depends explicitly on the forcing term). Explicit recursive equations that provide these two components are derived. It is shown that the feed-forward part makes a negligible contribution to the control signal. We also derive an explicit expression that a priori (i.e., before solving the control problem) leads to the minimum of the objective cost function (i.e., the fundamental performance limit), based only on the system matrices and the initial and free-stream boundary conditions. The adjoint equations admit a self-similar solution for large spanwise wave numbers with a scaling which is different from that of the LUBR equations. The controlled flow field also has a self-similar solution if the weighting matrices of the objective function are chosen appropriately. The code developed to implement this algorithm is efficient and has modest memory requirements. Computations show the significant reduction of energy for each wave number. The control of the full spectrum streaks, for conditions corresponding to a realistic experimental case, shows that the root-mean-square of the streamwise velocity is strongly suppressed in the whole domain and for all the frequency ranges examined.

DOI: 10.1103/PhysRevFluids.1.043501

\section{INTRODUCTION}

Active flow control involves the dynamic manipulation of a flow field through an actuation mechanism to achieve a desired objective. Feedback control theory applied to the Navier-Stokes equations provides a rigorous basis that can be exploited to effect a desired flow alteration, which can bring enormous environmental and economic benefits. This area has emerged as a major field of

\footnotetext{
*g.papadakis@ic.ac.uk
} 
research in fluid mechanics in the past 15 years or so, and thorough reviews have already appeared in the literature [1-5]. To set the context of the present contribution, we first briefly review work on control of disturbances in boundary layers.

Initial efforts to control shear flows applied control laws to the linearized Navier-Stokes equations for spatially invariant systems, such as channel flow. Along the homogeneous directions, the variation can be represented by Fourier series expansions, and the property of linearity allows the decoupling of the evolution equations for each wave number pair, thus greatly simplifying the control design [6-8]. Bamieh et al. [9] proved that, using this approach in spatially invariant systems with distributed actuation and sensing, spatially localized convolution kernels with exponential decay rates can be obtained. Such convolution kernels were found by Högberg and Henningson [10] using the Blasius mean velocity profile and were applied to boundary layer flows assuming full information of the flow field. Chevalier et al. [11] extended this work to account for stochastic disturbances that affect the system dynamics and corrupt wall measurements. Such disturbances, assumed to be white Gaussian noise, account, for example, for wall roughness, acoustic waves, or free-stream turbulence and were represented by a stochastic term on the right-hand side of an otherwise deterministic dynamical system. Measurements of skin friction and pressure (also affected by white noise) were used to estimate the state of the system using a Kalman filter. The separation principle allows solving the control and estimation problems separately and then combining them to form a compensator. This is the Linear Quadratic Gaussian (LQG) control approach and was employed in Chevalier et al. [11]. Estimation convolution kernels were also computed in this work.

Cathalifaud and Luchini [12] assumed homogeneity only in the spanwise direction and formulated a control minimization problem of the perturbation energy at a particular spanwise wave number using the linearized boundary layer equations as a constraint. The disturbances were assumed to be steady, and only the spatial evolution was considered. To solve the optimization problem, the authors derived a system of equations adjoint to the boundary layer equations and applied a forward-backward iterative approach to attain the optimal control actuation wall-normal velocity. They assumed full knowledge of the flow field and applied their method to flat and concave walls.

Cathalifaud and Bewley $[13,14]$ used the same disturbance evolution equations as Cathalifaud and Luchini [12] but took into account the system disturbances and modeled uncertainties by adding them to the right-hand side of the equations. However, they did not follow the LQG approach. Instead, they formulated a framework that incorporates directly the perturbations into the state vector. To achieve this, they augmented the state vector at each spatial location by appending the disturbances entering the system at all downstream locations. They derived the solution of the full-information control problem and expressed the actuation velocity as a function of the initial augmented state vector. They also solved the corresponding estimation problem. Assuming that the wall-shear stress and the pressure can be measured along the whole length of the wall, they obtained the best estimation of the initial augmented state vector, i.e., they solved the "smoothing problem" [15,16]. In Ref. [14] they demonstrated the success of their idea for perturbations introduced at the inlet.

More recent work on control of boundary layers dispenses entirely with the homogeneity assumption in any direction. This however leads to a very large number of states [of the order $O\left(10^{5}\right)-O\left(10^{6}\right)$ ] that renders the solution of the corresponding Riccati equation intractable. Therefore most of the recent work has focused on the application of low-order models that capture the input-output behavior of the system using orders of magnitude smaller number of states. Successful low-order models have been obtained using the balanced truncation method $[17,18]$. For example, Bagheri et al. [19] studied the feedback control of 2D perturbations in a boundary layer. The open loop system had approximately $10^{5}$ degrees of freedom, and a BPOD reduced-order model of order 50 was found to approximate the input-output behavior very well. The reduced order model was then used to design an LQG controller. This work was extended to three-dimensional perturbations in Semerano et al. [20]. Efforts have also been directed to avoiding the direct solution of the Riccati equation using the adjoint of the direct-adjoint methodology, proposed by Pralits and Luchini [21]. This approach bypasses the need for a reduced order model but requires full flow information. Recently Semerano et al. [22] showed that an analogous method can be applied for the estimation 


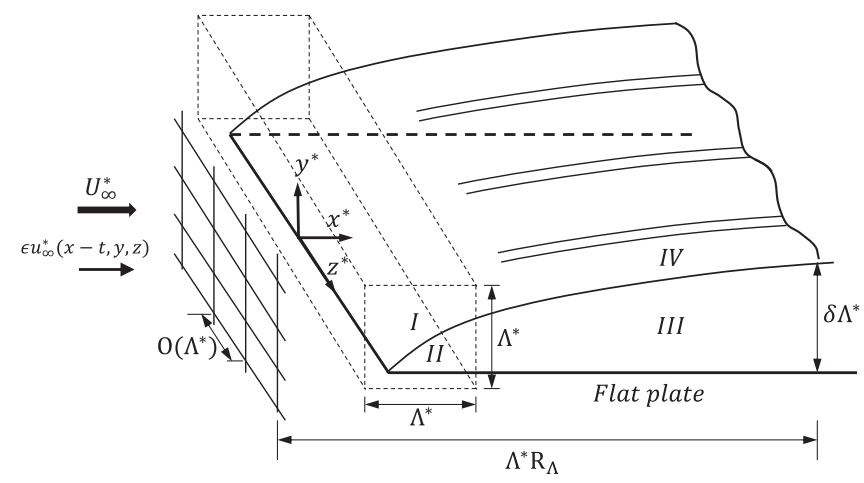

FIG. 1. Schematic of the flow domain illustrating the asymptotic structure of the boundary layer (adapted from Leib et al. [23]).

problem; the dual algorithm was referred to as adjoint of the adjoint-direct. By combining the solutions of the estimation and control problem, full-dimensional LQG controllers were obtained and used for the attenuation of the disturbances arising in two-dimensional boundary layer flows.

In all previous investigations the effect of free-stream turbulence is modeled as a stochastic term. It is assumed to be white Gaussian noise added to the right-hand side of an otherwise deterministic system. In the present paper we study the control problem of streaks generated inside a boundary layer due to free-stream turbulence theoretically and numerically using the framework of Leib et al. [23]. This framework describes the fundamental physical processes for the creation of streaks and directly incorporates the effect of randomness into the equations in a mathematically consistent way. This results in significant deviations in the formulation of the control problem compared to the standard approach used to deal with random perturbations.

Section II describes the mathematical framework of the physical problem, and Sec. III details the conversion of the model to a form suitable for control. The definition of the cost function and the formulation of the associated optimal control problem as well as the solution are presented in Secs. IV and V, respectively. Analysis of the differential form of the adjoint equations and the optimality condition leads to the identification of self-similar solutions, as explained in Sec. VI. Numerical results are presented and discussed in Sec. VII, and a summary of the main findings is found in Sec. VIII.

\section{MATHEMATICAL FORMULATION OF THE PHYSICAL PROBLEM}

In this section the mathematical description of the physical problem is presented. For more details, the reader is referred to Leib et al. [23]. We consider a flow of uniform velocity $U_{\infty}^{*}$ over an infinitely thin flat plate and a homogeneous, statistically stationary turbulence field away from the plate. The vortical perturbations are passively advected by the mean flow. The flow is described in a Cartesian coordinate system with position vector $\mathbf{x}=x \hat{\mathbf{i}}+y \hat{\mathbf{j}}+z \hat{\mathbf{k}}$, where $x, y$, and $z$ define the streamwise, wall-normal, and spanwise coordinates, respectively. These coordinates are scaled by $\Lambda^{*}$, the spanwise integral length scale of the free-stream turbulence. Quantities denoted by the superscript $*$ are dimensional. If turbulence is produced by a grid located upstream of the plate, then $\Lambda^{*}$ can be taken to represent the grid spacing, as shown in Fig. 1. Velocities and pressure are made dimensionless by $U_{\infty}^{*}$ and $\rho^{*} U_{\infty}^{* 2}$, respectively, where $\rho^{*}$ is the constant density of the fluid. Time is normalized by $\Lambda^{*} / U_{\infty}^{*}$.

Turbulence in the free stream is assumed to have low intensity, and therefore the velocity can be represented as a linear superposition of harmonic disturbances of the form

$$
\mathbf{u}=\hat{\mathbf{i}}+\epsilon \mathbf{u}_{\infty}(x-t, y, z)=\hat{\mathbf{i}}+\epsilon \hat{\mathbf{u}}^{\infty} e^{i\left(\mathbf{k} \cdot \mathbf{x}-k_{1} t\right)}+\text { c.c. },
$$


where $\epsilon$ is a measure of the turbulence intensity, $\hat{\mathbf{u}}^{\infty}=\left\{\hat{u}_{1}^{\infty}, \hat{u}_{2}^{\infty}, \hat{u}_{3}^{\infty}\right\}$ is the vector of random Fourier coefficients, $\mathbf{k}=\left\{k_{1}, k_{2}, k_{3}\right\}$ is the wave number vector, with components $k_{1}, k_{2}$, and $k_{3}$ in the streamwise, wall-normal, and spanwise directions, respectively, and c.c. represents the complex conjugate. We have considered only one Fourier mode. The instantaneous velocity is obtained by integration over all wave numbers; see Ref. [24]. Experiments of the Klebanoff modes in a pretransitional boundary layer clearly show that the perturbations are time periodic, and therefore Fourier decomposition is used in time.

In the above equation, $k_{1}$ is the scaled frequency, $k_{1}=2 \pi f_{1}^{*} \Lambda^{*} / U_{\infty}^{*}$, and is equal to the scaled streamwise wave number $k_{1}=2 \pi \Lambda^{*} / \lambda_{x}^{*}$, where $f_{1}^{*}$ is the dimensional frequency and $\lambda_{x}^{*}$ is the streamwise wavelength, respectively. These are equal because the free-stream perturbation is a convective vortical gust: it is of small amplitude (linear perturbation), and therefore the perturbation is convected at velocity $U_{\infty}^{*}$, i.e., $\lambda_{x}^{*}=U_{\infty}^{*} / f_{1}^{*}$ (Taylor's hypothesis).

Due to the incompressibility condition,

$$
\hat{\mathbf{u}}^{\infty} \cdot \mathbf{k}=0 .
$$

We define the Reynolds number as $R_{\Lambda} \equiv U_{\infty}^{*} \Lambda^{*} / \nu^{*}$, where $v^{*}$ is the kinematic viscosity of the fluid. A turbulent Reynolds number $r_{t}$ is defined as $r_{t}=\epsilon R_{\Lambda}$, which, at least initially, is of $O(1)$. When $R_{\Lambda}$ becomes asymptotically large [and therefore $\epsilon \rightarrow 0$ to keep $r_{t}$ fixed at $O(1)$ ] the flow domain can be divided into four asymptotic regions as shown in Fig. 1 [25].

Region I is an inviscid region which has $O\left(\Lambda^{*}\right)$ dimensions surrounding the leading edge of the plate. The flow is determined by generalized rapid distortion theory [26], and the velocities at the plate are

$$
\mathbf{u}=\hat{\mathbf{i}}+\epsilon\left(u_{1}^{(1)}, 0, u_{3}^{(1)}\right) e^{i\left[k_{1}(x-t)+k_{3} z\right]}+\text { c.c. }
$$

where $u_{1}^{(1)}=\hat{u}_{1}^{\infty}+\left(i k_{1} / \gamma\right) \hat{u}_{2}^{\infty}, u_{3}^{(1)}=\hat{u}_{3}^{\infty}+\left(i k_{3} / \gamma\right) \hat{u}_{2}^{\infty}$, and $\gamma=\sqrt{k_{1}^{2}+k_{3}^{2}}$.

Region II is a viscous region located underneath region I. The unsteady perturbations in this region are governed by the linearized unsteady boundary-layer (LUBL) equations [23,27]. These equations retain only the wall-normal viscous diffusion terms, while viscous diffusion in the spanwise direction is asymptotically smaller. As the boundary layer grows downstream, the LUBL equations become invalid when the boundary layer thickness becomes of the order of the spanwise length scale. This occurs in region III, which is the region of interest in this paper. The perturbation solution in region III is assumed to have the form [23]

$$
\begin{aligned}
\{\mathbf{u}, p\}= & \left\{F^{\prime}(\eta),\left(2 x R_{\Lambda}\right)^{-1 / 2}\left(\eta F^{\prime}-F\right), 0,-\frac{1}{2}\right\} \\
& +\epsilon\left\{\bar{u}_{0}(\bar{x}, \eta),\left(\frac{2 \bar{x} k_{1}}{R_{\Lambda}}\right)^{1 / 2} \bar{v}_{0}(\bar{x}, \eta), \bar{w}_{0}(\bar{x}, \eta), \bar{p}_{0}(\bar{x}, \eta)\right\} e^{i\left(k_{3} z-k_{1} t\right)},
\end{aligned}
$$

where $p$ is the pressure and $F$ denotes the solution of the Blasius equation for the mean flow, $F^{\prime \prime \prime}+F F^{\prime \prime}=0$, subject to the boundary conditions $F(0)=0, F^{\prime}(0)=0, F \rightarrow \bar{\eta}=\eta-\beta$, and $\beta=$ $1.217[28]$ as $\eta \rightarrow \infty$, where

$$
\eta=y\left(\frac{R_{\Lambda}}{2 x}\right)^{1 / 2}
$$

and $\bar{x}=k_{1} x$ is a scaled streamwise variable. We are interested in the evolution of perturbations with long wavelength only, so $k_{1} \ll 1$. The vector $\overline{\mathbf{q}}_{0}=\left\{\bar{u}_{0} \bar{v}_{0} \bar{w}_{0} \bar{p}_{0}\right\}^{T}$ is random and reflects the stochastic nature of turbulence. Its components can be decomposed in two parts as suggested in Ref. [29]: component $\{\bar{u}, \bar{v}, \bar{w}, \bar{p}\}$ and component $\left\{\bar{u}^{(0)}, \bar{v}^{(0)}, \bar{w}^{(0)}, \bar{p}^{(0)}\right\}$. The latter is dominant only in the outer part of the boundary layer [30] and is not considered here. The former component is driven by the random spanwise slip velocity $u_{3}^{(1)}=\hat{u}_{3}^{\infty}+\left(i k_{3} / \gamma\right) \hat{u}_{2}^{\infty}$ and is larger than the outer part by a 
factor $k_{3} / k_{1} \gg 1$. This component is retained in the present analysis:

$$
\left\{\bar{u}_{0}, \bar{v}_{0}, \bar{w}_{0}, \bar{p}_{0}\right\}=\left(\hat{u}_{3}^{\infty}+\frac{i k_{3}}{\gamma} \hat{u}_{2}^{\infty}\right)\left\{\frac{i k_{3}}{k_{1}} \bar{u}, \frac{i k_{3}}{k_{1}} \bar{v}, \bar{w}, i \kappa\left(\frac{k_{1}}{R_{\Lambda}}\right)^{1 / 2} \bar{p}\right\} .
$$

The randomness of free-stream turbulence (incorporated in the random Fourier coefficients $\hat{u}_{1}^{\infty}, \hat{u}_{2}^{\infty}, \hat{u}_{3}^{\infty}$ ) appears as a multiplicative factor of the deterministic variables $\{\bar{u}, \bar{v}, \bar{w}, \bar{p}\}$ (see Appendix A for more details). The equations for these variables can be obtained by substituting (4) and (6) into the Navier-Stokes equations. The equations can be linearized about the undisturbed Blasius solution when $r_{t} \ll 1$. For perturbations with long wavelength $\left(k_{1} \ll 1\right)$, the resulting equations take the form

$$
\begin{gathered}
-i \bar{u}+F^{\prime} \frac{\partial \bar{u}}{\partial \bar{x}}-\frac{F}{2 \bar{x}} \frac{\partial \bar{u}}{\partial \eta}-\frac{\eta F^{\prime \prime}}{2 \bar{x}} \bar{u}+F^{\prime \prime} \bar{v}=\frac{1}{2 \bar{x}} \frac{\partial^{2} \bar{u}}{\partial \eta^{2}}-\kappa^{2} \bar{u} \\
-i \bar{v}+F^{\prime} \frac{\partial \bar{v}}{\partial \bar{x}}-\frac{F}{2 \bar{x}} \frac{\partial \bar{v}}{\partial \eta}-\frac{1}{(2 \bar{x})^{2}}\left[\eta\left(\eta F^{\prime}\right)^{\prime}-F\right] \bar{u}+\frac{\left(\eta F^{\prime}\right)^{\prime}}{2 \bar{x}} \bar{v}=-\frac{1}{2 \bar{x}} \frac{\partial \bar{p}}{\partial \eta}+\frac{1}{2 \bar{x}} \frac{\partial^{2} \bar{v}}{\partial \eta^{2}}-\kappa^{2} \bar{v}, \\
-i \bar{w}+F^{\prime} \frac{\partial \bar{w}}{\partial \bar{x}}-\frac{F}{2 \bar{x}} \frac{\partial \bar{w}}{\partial \eta}=\kappa^{2} \bar{p}+\frac{1}{2 \bar{x}} \frac{\partial^{2} \bar{w}}{\partial \eta^{2}}-\kappa^{2} \bar{w}, \\
\frac{\partial \bar{u}}{\partial \bar{x}}-\frac{\eta}{2 \bar{x}} \frac{\partial \bar{u}}{\partial \eta}+\frac{\partial \bar{v}}{\partial \eta}+\bar{w}=0,
\end{gathered}
$$

where $\kappa$ is the scaled spanwise wave number, defined as $\kappa=k_{3} /\left(k_{1} R_{\Lambda}\right)^{1 / 2}=O(1)$. Equations (7)-(10) are called the linearized unsteady boundary-region (LUBR) equations and are the rational asymptotic limit of the Navier-Stokes equations for perturbations of low-frequency and low-streamwise wave number. Note that the spanwise viscous diffusion terms are retained in these equations. The steady form of this set was used, for example, in Refs. [12,13]. In symbolic form the system can be written as

$$
\mathcal{L} \frac{\partial \overline{\mathbf{q}}}{\partial \bar{x}}=\mathcal{M}(\overline{\mathbf{q}})
$$

where $\overline{\mathbf{q}}=\{\bar{u} \bar{v} \bar{w} \bar{p}\}^{T}$. The matrix $\mathcal{L}$ and the linear operator $\mathcal{M}$ can be obtained by inspection.

In summary, we have a deterministic parabolic system (11) from which the variables that contain the flow randomness $\left\{\bar{u}_{0}, \bar{v}_{0}, \bar{w}_{0}, \bar{p}_{0}\right\}$ are obtained using the multiplicative transformation (6) and not additively as usual. Of course, the objective function to be minimized by the control action must be expressed in terms of the original random variables $\left\{\bar{u}_{0}, \bar{v}_{0}, \bar{w}_{0}, \bar{p}_{0}\right\}$. This has important implications for the definition of the objective function and the solution, as discussed in Sec. IV.

These equations are solved by using initial and free-stream boundary conditions. The derivation of the initial boundary conditions is detailed in Ref. [23]. The free-stream conditions can be derived as $\eta \rightarrow \infty$ by considering the flow in region IV, which is above region III, as shown in Fig. 1 . The large- $\eta$ asymptotic solution of the LUBR equations is matched with the solution of region IV and provides the free-stream boundary conditions [23]:

$$
\begin{gathered}
\bar{u} \rightarrow 0, \\
\left\{\frac{\partial}{\partial \eta}+|\kappa|(2 \bar{x})^{1 / 2}\right\}\{\bar{v}, \bar{w}, \bar{p}\} \rightarrow\left\{-1, i \kappa_{2}(2 \bar{x})^{1 / 2}, 0\right\} e^{i\left[\bar{x}+\kappa_{2}(2 \bar{x})^{1 / 2} \bar{\eta}\right]-\left(\kappa^{2}+\kappa_{2}^{2}\right) \bar{x}},
\end{gathered}
$$

as $\eta \rightarrow \infty$, where $\kappa_{2}=k_{2} /\left(k_{1} R_{\Lambda}\right)^{1 / 2}=O(1)$ is the scaled wall-normal wave number.

The forcing on the right-hand side of (13) takes into account three factors: $e^{i \bar{x}}$ accounts for the downstream propagation of the disturbance (when combined with $\left.e^{-i k_{1} t}\right), e^{i \kappa_{2}(2 \bar{x})^{1 / 2} \bar{\eta}}$ accounts for the displacement effect of the mean boundary layer, and $e^{-\left(\kappa^{2}+\kappa_{2}^{2}\right) \bar{x}}$ accounts for the viscous decay of free-stream-turbulence in the streamwise direction. 


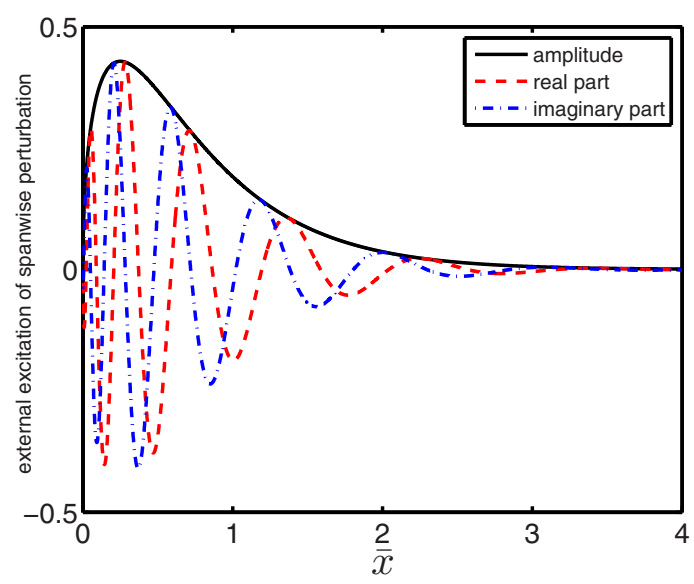

FIG. 2. Excitation term for $\bar{w}$ equation for $\kappa=1, \kappa_{2}=-1$.

The effect of wall-normal wave number $\kappa_{2}$ appears on the right-hand side of Eq. (13). It is instructive to examine how $\kappa_{2}$ affects these terms. For the $\bar{v}$ equation, the magnitude $e^{-\left(\kappa^{2}+\kappa_{2}^{2}\right) \bar{x}}$ peaks at the leading edge of the plate and decays exponentially; increasing $\kappa_{2}$ results in a faster viscous decay. For the $\bar{w}$ equation, the magnitude $\kappa_{2}(2 \bar{x})^{1 / 2} e^{-\left(\kappa^{2}+\kappa_{2}^{2}\right) \bar{x}}$ is 0 at the leading edge and peaks at location $\bar{x}_{\min }=0.5\left(\kappa^{2}+\kappa_{2}^{2}\right)^{-1}$ (see Fig. 2) before decaying. Increasing $\kappa_{2}$ brings the peak closer to the leading edge and results in a faster viscous decay. These observations will be used later to explain the effect of $\kappa_{2}$ on the controlled flow field.

The boundary conditions for the velocity components can be used to obtain conditions at the free stream for the three vorticity components. Substituting the flow decomposition (4) and (6) into the vorticity vector definition $\omega_{i}(i=x, y, z)$ and simplifying by taking into account that $k_{1} \ll 1$, it is found that $\omega_{i}=\epsilon\left(\hat{u}_{3}^{\infty}+i k_{3} \hat{u}_{2}^{\infty} / \gamma\right) \bar{\omega}_{i} e^{i\left(k_{3} z-k_{1} t\right)}$ where

$$
\begin{gathered}
\bar{\omega}_{x}=\left(R_{\Lambda} k_{1}\right)^{1 / 2}\left[\frac{1}{(2 \bar{x})^{1 / 2}} \frac{\partial \bar{w}}{\partial \eta}+\kappa^{2}(2 \bar{x})^{1 / 2} \bar{v}\right], \\
\bar{\omega}_{y}=-\kappa^{2} R_{\Lambda} \bar{u} \\
\bar{\omega}_{z}=-\frac{i \kappa R_{\Lambda}}{(2 \bar{x})^{1 / 2}} \frac{\partial \bar{u}}{\partial \eta} .
\end{gathered}
$$

The components $\bar{\omega}_{y}$ and $\bar{\omega}_{z}$ are proportional to $\bar{u}$ and $\frac{\partial \bar{u}}{\partial \eta}$, respectively, and are both 0 in the free stream. On the other hand, $\bar{\omega}_{x}$ is sensitive to the free-stream forcing. Indeed, it is possible to find an explicit expression for the scaled streamwise vorticity, $\bar{\omega}_{x} /\left(R_{\Lambda} k_{1}\right)^{1 / 2}$, in the free stream. The process to derive this expression is the following: multiply the condition for $\bar{v}$ by $|\kappa|(2 \bar{x})^{1 / 2}$, add the result to the condition for $\bar{w}$, and simplify by taking into account that $\bar{w}+\frac{\partial \bar{v}}{\partial \eta}=0$ in the free stream [the later is due to the continuity equation (10) and the fact that $\bar{u}=\frac{\partial \bar{u}}{\partial \eta}=0$ from Eq. (12)]. The final expression is

$$
\frac{\left.\bar{\omega}_{x}\right|_{\eta \rightarrow \infty}}{\left(R_{\Lambda} k_{1}\right)^{1 / 2}}=\left(i \kappa_{2}-|\kappa|\right) e^{i\left[\bar{x}+\kappa_{2}(2 \bar{x})^{1 / 2} \bar{\eta}\right]-\left(\kappa^{2}+\kappa_{2}^{2}\right) \bar{x}} .
$$

The boundary conditions on velocities [Eqs. (12) and (13)] therefore result in injection of streamwise vorticity at the top of the boundary layer. As will be discussed in Sec. VII, this vorticity penetrates inside the boundary layer. 


\section{CONVERSION OF THE MODEL TO A FORM SUITABLE FOR CONTROL}

Equations (7)-(10), together with the associated boundary and initial conditions, form the mathematical model of the physical system for which the control will be designed. The implementation of wall transpiration follows the boundary lifting approach [10]. In the uncontroled case, the system (11) satisfies the no-slip boundary condition at the wall. Flow actuation through wall transpiration introduces an inhomogeneous boundary condition. Since the system is linear, the state vector $\overline{\mathbf{q}}$ can be written as the sum of two parts: $\mathbf{q}_{h}$, the solution of the homogeneous problem with homogeneous boundary condition, and $\mathbf{q}_{p}$, the solution of the homogeneous problem with inhomogeneous boundary condition [10]. This can be expressed as

$$
\overline{\mathbf{q}}=\mathbf{q}_{h}+\bar{v}_{w}(\bar{x}) \mathbf{q}_{p},
$$

where $\bar{v}_{w}(\bar{x})$ is the wall-normal actuation velocity and $\mathbf{q}_{p}$ is the particular solution of the homogeneous system when the wall-normal velocity is set to $\bar{v}_{w}(\bar{x})=1$. Substitution of (18) into (11) leads to

$$
\mathcal{L} \frac{\partial\left(\mathbf{q}_{h}+\bar{v}_{w}(\bar{x}) \mathbf{q}_{p}\right)}{\partial \bar{x}}=\mathcal{M}\left(\mathbf{q}_{h}+\bar{v}_{w}(\bar{x}) \mathbf{q}_{p}\right)
$$

If $\mathbf{q}_{p}$ is selected to satisfy

$$
\mathcal{L} \frac{\partial \mathbf{q}_{p}}{\partial \bar{x}}=\mathcal{M} \mathbf{q}_{p}
$$

the system (19) becomes

$$
\mathcal{L} \frac{\partial \mathbf{q}_{h}}{\partial \bar{x}}=\mathcal{M} \mathbf{q}_{h}-\mathcal{L} \mathbf{q}_{p} \frac{d \bar{v}_{w}(\bar{x})}{d \bar{x}} .
$$

Defining the augmented state vector

$$
\tilde{\mathbf{q}}=\left[\begin{array}{l}
\mathbf{q}_{h} \\
\bar{v}_{w}
\end{array}\right]
$$

one finds

$$
\mathcal{L}_{a} \frac{\partial \tilde{\mathbf{q}}}{\partial \bar{x}}=\mathcal{M}_{a} \tilde{\mathbf{q}}+\left[\begin{array}{c}
-\mathcal{L} \mathbf{q}_{p} \\
1
\end{array}\right] \frac{d \bar{v}_{w}(\bar{x})}{d \bar{x}},
$$

where $\mathcal{L}_{a}=\left[\begin{array}{cc}\mathcal{L} & 0 \\ 0 & 1\end{array}\right]$ and $\mathcal{M}_{a}=\left[\begin{array}{cc}\mathcal{M} & 0 \\ 0 & { }_{0}\end{array}\right]$ are the augmented matrix $\mathcal{L}$ and operator $\mathcal{M}$, respectively.

The system (23) is first discretized in the wall-normal direction $\eta$ using rational Chebyshev polynomials (see Appendix B for details). The resulting semidiscrete system in matrix form is

$$
\left[\begin{array}{ll}
\mathbf{L} & 0 \\
0 & 1
\end{array}\right] \frac{d \mathbf{q}}{d \bar{x}}=\left[\begin{array}{cc}
\mathbf{M} & 0 \\
0 & 0
\end{array}\right] \mathbf{q}+\left[\begin{array}{c}
-\mathbf{L} \mathbf{q}_{p} \\
1
\end{array}\right] \frac{d \bar{v}_{w}(\bar{x})}{d \bar{x}}+\left[\begin{array}{c}
\mathbf{G} \\
0
\end{array}\right]
$$

where $\mathbf{q}=\left[\begin{array}{l}\hat{\mathbf{q}}_{h} \\ \bar{v}_{w}\end{array}\right]$ and $\hat{\mathbf{q}}_{h}$ is the vector of the coefficients of Chebyshev polynomials for $\mathbf{q}_{h}$, while matrices $\mathbf{L}, \mathbf{M}$ are the discrete forms of $\mathcal{L}_{a}$ and $\mathcal{M}_{a}$, respectively. The vector $\mathbf{G}(\bar{x})$ represents the discrete form of the forcing, i.e., the right-hand side of expressions (12) and (13).

This system is parabolic in the streamwise direction, $\bar{x}$. Because the streamwise derivative of pressure does not appear in the equations, matrix $\mathbf{L}$ is singular and cannot be inverted. In the control community, such linear systems are called "descriptor systems" [31]. To avoid the special treatment that such systems require, the following approach is applied. An implicit finite difference discretization in the streamwise direction is employed to convert the continuous system to a discrete system. With first-order Euler implicit scheme and after some rearrangement, system (24) becomes

$$
\left\{\left[\begin{array}{cc}
\mathbf{L}_{i} & 0 \\
0 & 1
\end{array}\right] \frac{1}{\Delta \bar{x}}-\left[\begin{array}{cc}
\mathbf{M}_{i} & 0 \\
0 & 0
\end{array}\right]\right\} \mathbf{q}_{i+1}=\left[\begin{array}{cc}
\mathbf{L}_{i} & 0 \\
0 & 1
\end{array}\right] \frac{1}{\Delta \bar{x}} \mathbf{q}_{i}+\left[\begin{array}{c}
-\mathbf{L}_{i} \mathbf{q}_{p_{i}} \\
1
\end{array}\right] \frac{d \bar{v}_{w}(\bar{x})}{d \bar{x}}+\left[\begin{array}{c}
\mathbf{G}_{i} \\
0
\end{array}\right]
$$


where the indices $i+1$ and $i$ refer to variables at positions $\bar{x}_{i+1}$ and $\bar{x}_{i}$ respectively, and $\Delta \bar{x}=$ $\bar{x}_{i+1}-\bar{x}_{i}$. A second-order backward approximation could have been employed, but this would have resulted in double the number of state variables. With small enough $\Delta \bar{x}$, a first-order approximation provides accurate results, as shown in Appendix B. The presence of the factor $1 / \bar{x}$ into the equations can lead to poor conditioning as $\Delta \bar{x}$ is reduced. To avoid this, we have multiplied left- and right-hand sides with $\bar{x}$ and then discretized the equations.

The matrix on the left-hand side is now invertible, and the unknown variables at the new streamwise position can be obtained from

$$
\mathbf{q}_{i+1}=\mathbf{A}_{i} \mathbf{q}_{i}+\mathbf{B}_{i} u_{i}+\mathbf{C}_{i},
$$

where $u_{i}=\bar{v}_{w}^{\prime}(\bar{x})$ and the prime here indicates differentiation. Matrix $\mathbf{A}_{i}$ is

$$
\mathbf{A}_{i}=\left\{\left[\begin{array}{cc}
\mathbf{L}_{i} & 0 \\
0 & 1
\end{array}\right] \frac{1}{\Delta \bar{x}}-\left[\begin{array}{cc}
\mathbf{M}_{i} & 0 \\
0 & 0
\end{array}\right]\right\}^{-1}\left[\begin{array}{cc}
\mathbf{L}_{i} & 0 \\
0 & 1
\end{array}\right] \frac{1}{\Delta \bar{x}}
$$

with similar expressions for matrices $\mathbf{B}_{i}$ and $\mathbf{C}_{i}$. The standard theory of linear discrete-time control systems can now be directly applied, where the role of time is replaced by the parabolic direction $\bar{x}$. It can be readily shown that the vectors $\overline{\mathbf{q}}$ and $\tilde{\mathbf{q}}$ are related by

$$
\overline{\mathbf{q}}=\left[\begin{array}{ll}
\mathbf{I}_{4 \times 4} & \mathbf{q}_{p}
\end{array}\right] \tilde{\mathbf{q}},
$$

where $\mathbf{I}_{4 \times 4}$ is the identity matrix of order four.

\section{DEFINITION OF THE COST FUNCTION AND FORMULATION OF THE OPTIMAL CONTROL PROBLEM}

It is reasonable to define the cost function as the integral of the perturbation kinetic energy in the whole domain:

$$
E=\frac{1}{2} \int_{\bar{x}_{0}}^{\bar{x}_{f}} \int_{0}^{\infty}\left\langle u^{\prime 2}\right\rangle d \eta d x
$$

where $\left\langle u^{\prime 2}\right\rangle$ is the mean-square fluctuation of the streamwise velocity and \langle\rangle denotes the expectation operator. The contribution of the other two velocity components is neglected because it is asymptotically smaller as $k_{1} \ll 1$.

The linearity of the equations can be used to obtain the distribution of the statistical quantity $\left\langle u^{\prime 2}\right\rangle(x, \eta)$ inside the boundary layer. Using (4) and superposing the contributions from all wave numbers, the instantaneous velocity can be written as

$$
u(x, \eta, z, t)=\int_{-\infty}^{+\infty} \int_{-\infty}^{+\infty} \int_{-\infty}^{+\infty} \bar{u}_{0}\left(k_{1} x, \eta\right) e^{i\left(k_{3} z-k_{1} t\right)} d k_{1} d k_{2} d k_{3} .
$$

The streamwise velocity component in Eq. (6) links the random fluctuating Fourier coefficients of the upstream turbulence to the fluctuating streamwise velocity inside the boundary layer, leading to

$$
u(x, \eta, z, t)=\int_{-\infty}^{+\infty} \int_{-\infty}^{+\infty} \int_{-\infty}^{+\infty} \frac{i k_{3}}{k_{1}}\left(\hat{u}_{3}^{\infty}+\frac{i k_{3}}{\gamma} \hat{u}_{2}^{\infty}\right) \bar{u}\left(k_{1} x, \eta\right) e^{i\left(k_{3} z-k_{1} t\right)} d k_{1} d k_{2} d k_{3} .
$$

A similar expression can be written for the velocity at any other point $\left(x^{\prime}, \eta^{\prime}, z^{\prime}\right)$ at a different time instant $\left(t^{\prime}\right)$ with $\left(k_{1}^{\prime}, k_{2}^{\prime}, k_{3}^{\prime}\right)$ as the running variables of the integrals. Multiplying the two expressions, taking the ensemble average, and using the property of the upstream turbulence spectral tensor, $\Phi_{\infty i j}(\mathbf{k}),\left\langle\hat{u}_{i}^{\infty}(\mathbf{k}) \hat{u}_{j}^{\infty \star}\left(\mathbf{k}^{\prime}\right)\right\rangle=\Phi_{\infty i j}(\mathbf{k}) \delta\left(\mathbf{k}-\mathbf{k}^{\prime}\right)$ (where a star hereafter denotes the conjugate transpose), explicit expressions for the two-point, time-delayed, correlations can be obtained (see Ref. [32]). Assuming that the two points coincide and there is no time delay, it is 
found that

$$
\left\langle u^{\prime 2}\right\rangle(x, \eta)=\int_{-\infty}^{+\infty} \int_{-\infty}^{+\infty} \int_{-\infty}^{+\infty} \frac{k_{3}^{2}}{k_{1}^{2}} \Phi_{t}|\bar{u}|^{2} d k_{1} d k_{2} d k_{3},
$$

where $\Phi_{t}=\Phi_{\infty 22}+\Phi_{\infty 33}$ is the transverse upstream velocity spectrum. Expression (32) contains the wave numbers $k_{1}, k_{3}$ (not the scaled wave numbers $\kappa, \kappa_{2}$ ) and, in order to compute the integral, a transformation of variables is necessary. See Refs. [23,33] for a detailed account of this transformation. Introducing the polar coordinates $k_{3}=k_{\perp} \sin \theta$ and $k_{2}=k_{\perp} \cos \theta$ and the new integration variable

$$
s=k_{1} R_{\Lambda} / k_{\perp}^{2}=\sin ^{2} \theta / \kappa^{2}
$$

Eq. (32) becomes

$$
\left\langle u^{\prime 2}\right\rangle(x, \eta)=R_{\Lambda} \int_{0}^{\infty} \Phi_{t}\left(0, k_{\perp}\right) K\left(k_{\perp} \bar{\delta}, \eta\right) k_{\perp} d k_{\perp},
$$

where $\bar{\delta}=\left(2 x / R_{\Lambda}\right)^{1 / 2}$ and $K\left(k_{\perp} \bar{\delta}, \eta\right)$ is the kernel function given by

$$
K\left(k_{\perp} \bar{\delta}, \eta\right)=2 \int_{0}^{\infty} \int_{0}^{2 \pi} \frac{\left|\kappa^{2} \bar{u}\right|^{2}}{\sin ^{2} \theta} d \theta d s
$$

Substituting (35) into (29), one finds

$$
E=\frac{R_{\Lambda}}{2} \int_{\bar{x}_{0}}^{\bar{x}_{f}} \int_{0}^{\infty} \int_{0}^{\infty} \int_{0}^{\infty} \int_{0}^{2 \pi} 2 \frac{\Phi_{t}\left(0, k_{\perp}\right)}{\sin ^{2} \theta}\left|\kappa^{2} \bar{u}\right|^{2} k_{\perp} d \theta d s d k_{\perp} d \eta d x
$$

and, swapping the orders of integration in the wave number and physical space, we finally obtain

$$
E=2 R_{\Lambda} \int_{0}^{\infty} \int_{0}^{\infty} \int_{0}^{2 \pi} \frac{\Phi_{t}\left(0, k_{\perp}\right) \kappa^{2}}{\sin ^{2} \theta}\left(\int_{\bar{x}_{0}}^{\bar{x}_{f}} \int_{0}^{\infty} \frac{1}{2} \kappa^{2}|\bar{u}|^{2} d \eta d x\right) k_{\perp} d \theta d s d k_{\perp} .
$$

This is the cost function to be minimized.

A few comments are due. In the standard control problem, the input disturbances are assumed to be white Gaussian noise and are added to the right-hand side of an otherwise deterministic system (see Ref. [34]). The objective is then the minimization of the $H_{2}$ norm that maps the random input disturbances to the energy of the output (plus the energy of the control effort). A random signal is called white noise if the correlation between any two time instances, $t$ and $\tau$, is proportional to the delta function, $\delta(t-\tau)$. It is reminded that the $H_{2}$ norm of a system is the expected root-mean-square value of the output when the input is a unit-variance white noise process.

In the present context, the cost function is defined as an expectation obtained by integrating over the wave number space. The property $\left\langle\hat{u}_{i}^{\infty}(\mathbf{k}) \hat{u}_{j}^{\infty \star}\left(\mathbf{k}^{\prime}\right)\right\rangle=\Phi_{\infty i j}(\mathbf{k}) \delta\left(\mathbf{k}-\mathbf{k}^{\prime}\right)$ naturally introduces the spectral tensor of free-stream turbulence into the cost function. It is analogous to the property $\langle w(t) w(\tau)\rangle=\delta(t-\tau)$ of white noise employed in the standard control problem mentioned above. While the former is defined in the wave number space, the latter is defined in time. Both, however, when integrated in their corresponding spaces, provide the expectation of the random quadratic cost function that the control action will minimize.

It is clear then that the present formulation, directly based on a physically correct model of the flow and the evaluation of the energy over integration in wave number space, introduces the spectrum of turbulence into the control objective directly. It is also evident that the control problem makes sense only when solved for all wave numbers because it is only then that the expectation (that is a quantity that has physical meaning, like $\left\langle u^{\prime 2}\right\rangle$ ) can be properly defined.

Equation (37) makes clear that, in order to minimize the cost function, we have to minimize the energy at each wave number in the whole domain. As discussed in Sec. VI, due to asymptotic self-similar behavior of the particular solution $\mathbf{q}_{p}$ for large $\kappa$, we minimize the product $\kappa^{2}|\bar{u}|^{2} / 2$ 
instead of $|\bar{u}|^{2} / 2$. Note also that the spectrum $\Phi_{t}\left(0, k_{\perp}\right)$ is independent of the spatial variables and was factored out of the spatial integral. Therefore the spectrum of the upstream turbulence does not enter directly into the solution of the control problem at each wave number. In this sense, the controllers that will be derived are universal because the gains are independent of the spectrum.

In the following, the minimization problem of the energy for each wave number is examined. For each wave number vector $\mathbf{k}=\left(k_{1}, k_{2}, k_{3}\right)$ this energy is defined as

$$
\bar{E}(\mathbf{k})=\frac{1}{2} \int_{\bar{x}_{0}}^{\bar{x}_{f}} \int_{0}^{\infty} \kappa^{2}|\bar{u}(\bar{x}, \eta)|^{2} d \eta d \bar{x} .
$$

This follows directly from (37), the only difference being the integral running variable, i.e., $\bar{x}=k_{1} x$ instead of $x$. This is of course not a problem as $k_{1}$ is constant when the integration is performed inside the brackets in Eq. (37), and it is convenient because the equations for $\bar{u}$ are written in terms of $\bar{x}, \eta$. Note that the velocity $\bar{u}(x, \eta)$ asymptotically tends to 0 as $\eta \rightarrow \infty$ [from boundary condition (12)] and the inner integral in Eq. (38) is convergent. If we define

$$
\bar{E}(\mathbf{k})_{i}=\frac{1}{2} \int_{0}^{\infty} \kappa^{2}\left|\bar{u}\left(\bar{x}_{i}, \eta\right)\right|^{2} d \eta,
$$

then the discrete form of (38) becomes

$$
\bar{E}(\mathbf{k})=\Delta \bar{x} \sum_{i=0}^{N-1} \bar{E}(\mathbf{k})_{i} .
$$

The control objective for each wave number $\mathbf{k}$ is defined as

$$
J=\frac{1}{2} \mathbf{q}_{N}^{\star} \mathbf{P}_{N} \mathbf{q}_{N}+\frac{1}{2} \sum_{i=0}^{N-1}\left(\mathbf{q}_{i}^{\star} \mathbf{Q}_{i} \mathbf{q}_{i}+\mathbf{u}_{i}^{\star} \mathbf{R}_{i} \mathbf{u}_{i}\right),
$$

where the matrix $\mathbf{Q}_{i}$ is chosen such that $\mathbf{q}_{i}^{\star} \mathbf{Q}_{i} \mathbf{q}_{i}=\bar{E}(\mathbf{k})_{i}$. Matrices $\mathbf{Q}_{i}, \mathbf{R}_{i}$, and $\mathbf{P}_{N}$ are Hermitian weighting matrices. The matrix $\mathbf{R}_{i}$ penalizes the control effort while $\mathbf{P}_{N}$ penalizes the states at the end of the domain. Matrix $\mathbf{Q}_{i}$ is computed using the standard approach described in Refs. [10,35]. If matrix $\mathbf{Q}_{i}^{a}$ is such that $\bar{E}(\mathbf{k})_{i}=\overline{\mathbf{q}}_{i}^{\star} \mathbf{Q}_{i}^{a} \overline{\mathbf{q}}_{i}$, then use of (28) shows that $\bar{E}(\mathbf{k})_{i}=\mathbf{q}_{i}^{\star} \mathbf{Q}_{i} \mathbf{q}_{i}$, where

$$
\mathbf{Q}_{i}=\left[\begin{array}{cc}
\mathbf{Q}_{i}^{a} & \mathbf{Q}_{i}^{a} \mathbf{q}_{p} \\
\mathbf{q}_{p}^{\star} \mathbf{Q}_{i}^{a} & \mathbf{q}_{p}^{\star} \mathbf{Q}_{i}^{a} \mathbf{q}_{p}
\end{array}\right] .
$$

The matrix $\mathbf{Q}_{i}^{a}$ contains the weights from the numerical computation of the integral (39) along the $\eta$ direction. Only the diagonal submatrix that acts on the Chebyshev coefficients of $\bar{u}$ velocity is nonzero.

The developed method penalizes both the control velocity $\bar{v}_{w}$ [through term $\mathbf{q}_{p}^{\star} \mathbf{Q}_{i}^{a} \mathbf{q}_{p}$ in matrix (42)] and the streamwise derivative $u_{i}=\bar{v}_{w}^{\prime}(\bar{x})$ [through term $\mathbf{u}_{i}^{\star} \mathbf{R}_{i} \mathbf{u}_{i}$ in Eq. (41)]. This leads to a smooth variation of the control signal. It is possible to add extra penalty on $\bar{v}_{w}^{2}$ by multiplying $\mathbf{q}_{p}^{\star} \mathbf{Q}_{i}^{a} \mathbf{q}_{p}$ by a positive constant greater than 1 , but this was found to be unnecessary.

\section{SOLUTION OF THE OPTIMAL CONTROL PROBLEM}

The most important challenge for the synthesis of the controller is the presence of the known forcing term on the right-hand side of Eq. (26). This term necessitates the formulation and derivation of the solution of the control problem from first principles. In the present study, the theory of optimal state feedback control [16] will be used to minimize the quadratic cost function (41).

The objective is to find the control sequence $\mathbf{u}_{0}, \mathbf{u}_{1}, \ldots, \mathbf{u}_{N-1}$ that minimizes $J$. In order to find the solution for this optimization problem, we proceed as described in Refs. $[15,36]$ and consider 
the Hamiltonian function

$$
H_{i}=\frac{1}{2}\left(\mathbf{q}_{i}^{\star} \mathbf{Q}_{i} \mathbf{q}_{i}+\mathbf{u}_{i}^{\star} \mathbf{R}_{i} \mathbf{u}_{i}\right)+\lambda_{i+1}^{\star}\left(\mathbf{A}_{i} \mathbf{q}_{i}+\mathbf{B}_{i} \mathbf{u}_{i}+\mathbf{C}_{i}\right),
$$

where $\lambda_{i+1}^{\star}$ is the adjoint (or costate) variable. The equations for the state and adjoint variables are

$$
\begin{gathered}
\mathbf{q}_{i+1}=\frac{\partial H_{i}}{\partial \boldsymbol{\lambda}_{i+1}}=\mathbf{A}_{i} \mathbf{q}_{i}+\mathbf{B}_{i} \mathbf{u}_{i}+\mathbf{C}_{i}, \\
\lambda_{i}=\frac{\partial H_{i}}{\partial \mathbf{q}_{i}}=\mathbf{Q}_{i} \mathbf{q}_{i}+\mathbf{A}_{i}^{\star} \lambda_{i+1},
\end{gathered}
$$

respectively, and the stationarity (or optimality) condition is

$$
0=\frac{\partial H_{i}}{\partial \mathbf{u}_{i}}=\mathbf{B}_{i}^{\star} \lambda_{i+1}+\mathbf{R}_{i} \mathbf{u}_{i}
$$

from which

$$
\mathbf{u}_{i}=-\mathbf{R}_{i}^{-1} \mathbf{B}_{i}^{\star} \lambda_{i+1}
$$

The terminal condition for the adjoint equation at the end of the domain is

$$
\lambda_{N}=\frac{\partial}{\partial \mathbf{q}_{N}}\left(\frac{1}{2} \mathbf{q}_{N}^{\star} \mathbf{P}_{N} \mathbf{q}_{N}\right)=\mathbf{P}_{N} \mathbf{q}_{N} .
$$

Substituting (47) into (44) and putting the resulting equation together with (45) in matrix form yields the following inhomogeneous (i.e., forced) Hamiltonian system:

$$
\left[\begin{array}{c}
\mathbf{q}_{i+1} \\
\lambda_{i}
\end{array}\right]=\left[\begin{array}{cc}
\mathbf{A}_{i} & -\mathbf{B}_{i} \mathbf{R}_{i}^{-1} \mathbf{B}_{i}^{\star} \\
\mathbf{Q}_{i} & \mathbf{A}_{i}^{\star}
\end{array}\right]\left[\begin{array}{c}
\mathbf{q}_{i} \\
\lambda_{i+1}
\end{array}\right]+\left[\begin{array}{c}
\mathbf{C}_{i} \\
\mathbf{0}
\end{array}\right] .
$$

An inhomogeneous Hamiltonian system also appears in the formulation of linear quadratic tracking problems, where the output is required to follow a known reference signal over the interval $[0, N]$ [36]. However, for such problems the forcing appears in the adjoint equation and not in the state equation. Therefore, the solution of the optimal control problem must be rederived.

It is possible to obtain the solution of the system of Eqs. (44)-(46) iteratively. We start by guessing the wall-based control sequence $\mathbf{u}_{i}$, march forward Eq. (44), apply the terminal condition (48), march backward Eq. (45), compute the control variable from (47), and repeat the forward-backward marching until convergence. This is the approach followed in Ref. [12]. However, we show here that it is possible to obtain the solution by marching only once in the backward direction. Furthermore, the solution is given by closed form analytic expressions that clearly identify the contribution of the forcing.

To obtain such a solution to the standard Linear Quadratic Regulator (LQR) problem, we assume that there is a matrix $\mathbf{P}_{i}$ such as $\lambda_{i}=\mathbf{P}_{i} \mathbf{q}_{i}$ and a Riccati equation is constructed from which $\mathbf{P}_{i}$ is obtained (this is the well-known sweep method and is described in detail in Ref. [15]). The presence of the forcing term $\mathbf{C}_{i}$ in Eq. (49) precludes the use of the same expression to link the state and adjoint variables, $\mathbf{q}_{i}$ and $\lambda_{i}$. However, we can modify the sweep method and assume

$$
\lambda_{i}=\mathbf{P}_{i} \mathbf{q}_{i}-\mathbf{V}_{i}
$$

for some yet unknown sequences $\mathbf{P}_{i}$ and $\mathbf{V}_{i}$. The minus sign (-) in front of $\mathbf{V}_{i}$ in Eq. (50) is not important. It could have been a plus (+), but we have retained the minus sign as in Lewis et al. [36]. If consistent expressions can be found for $\mathbf{P}_{i}$ and $\mathbf{V}_{i}$, then this is a valid assumption. Note that the same assumption was also used to derive the optimal control for the tracking problem. As shown by the derivation in Appendix C, consistent expressions can indeed be found for our problem too, but the resulting equations are different since the forcing appears in the state, not the costate equation. 
The optimal control signal is

$$
\mathbf{u}_{i}=-\mathbf{K}_{i} \mathbf{q}_{i}+\mathbf{K}_{i}^{v}\left(\mathbf{V}_{i+1}-\mathbf{P}_{i+1} \mathbf{C}_{i}\right)
$$

where $\mathbf{K}_{i}$ and $\mathbf{K}_{i}^{v}$ are the gains,

$$
\begin{gathered}
\mathbf{K}_{i}=\left(\mathbf{R}_{i}+\mathbf{B}_{i}^{\star} \mathbf{P}_{i+1} \mathbf{B}_{i}\right)^{-1} \mathbf{B}_{i}^{\star} \mathbf{P}_{i+1} \mathbf{A}_{i}, \\
\mathbf{K}_{i}^{v}=\left(\mathbf{B}_{i}^{\star} \mathbf{P}_{i+1} \mathbf{B}_{i}+\mathbf{R}_{i}\right)^{-1} \mathbf{B}_{i}^{\star},
\end{gathered}
$$

$\mathbf{P}_{i}$ is found through the Riccati equation

$$
\mathbf{P}_{i}=\mathbf{A}_{i}^{\star} \mathbf{P}_{i+1}\left(\mathbf{A}_{i}-\mathbf{B}_{i} \mathbf{K}_{i}\right)+\mathbf{Q}_{i},
$$

and $\mathbf{V}_{i}$ is obtained as

$$
\mathbf{V}_{i}=\left(\mathbf{A}_{i}-\mathbf{B}_{i} \mathbf{K}_{i}\right)^{\star}\left(\mathbf{V}_{i+1}-\mathbf{P}_{i+1} \mathbf{C}_{i}\right)
$$

Equation (51) demonstrates that the control velocity consists of two components: a feedback component ( $-\mathbf{K}_{i} \mathbf{q}_{i}$ ) whose gain is dependent on the solution to the Riccati equation (54) and a second component $\left[\mathbf{K}_{i}^{v}\left(\mathbf{V}_{i+1}-\mathbf{P}_{i+1} \mathbf{C}_{i}\right)\right.$, which we call feed forward], whose gain is dependent on the auxiliary sequence $\mathbf{V}_{i}$, governed by Eq. (55). Note that the Riccati equation is independent of the forcing.

The feed-forward component depends entirely on the term $\mathbf{C}_{i}$. In the absence of this term, Eq. (C13) and the terminal condition (C15) yield $\mathbf{V}_{i}=0$ everywhere. In such a case, the feed-forward term is also 0 and, as expected, (51) yields the standard LQR solution. Note that the solution naturally accounts for the dependence of feedback and feed-forward gains on the streamwise location $\bar{x}_{i}$.

It is also possible to derive an analytic expression for the performance limit (i.e., the minimum value $J_{\min }$ of the cost function $J$ ) a priori, that is using only the matrices of the system and without computing first the optimal sequences $\mathbf{q}_{i}$ and $\mathbf{u}_{i}$. The derivation of this expression is given in Appendix D. The physical meaning of the sequence $\mathbf{V}_{i}$ is elucidated therein. It is proven that $-\mathbf{V}_{i}$ is the adjoint of the closed loop plant with respect to an appropriate cost function. The expression for $J_{\min }$ is useful because it separates analytically the effect of feedback and feed-forward components of the control signal. This is examined later in Sec. VII.

\section{DIFFERENTIAL FORM OF THE ADJOINT EQUATIONS AND SELF-SIMILAR FORM OF THE SOLUTION}

The previous section focused on the analytic solution of the control problem. The discrete adjoint equations were obtained directly from the discrete form of the direct problem. This is known as the adjoint of the discretization [37]. Since it leads directly to the discrete form of the adjoint equations (45), it hides important physical properties of the full direct-adjoint system that can be revealed only by examining the differential form of the equations. In this section the differential form of the adjoint equations is derived, and it is proven that, under certain conditions, the adjoint and the controlled field have self-similar behavior for large $\kappa$.

The derivation of the differential form follows the approach described in Ref. [38]. System (23) can be written as

$$
\mathcal{L}_{a} \frac{\partial \tilde{\mathbf{q}}}{\partial \bar{x}}=\mathbf{M}_{0} \tilde{\mathbf{q}}+\mathbf{M}_{1} \frac{\partial \tilde{\mathbf{q}}}{\partial \eta}+\mathbf{M}_{2} \frac{\partial^{2} \tilde{\mathbf{q}}}{\partial \eta^{2}}+\mathbf{M}_{3} \mathbf{u}
$$

where $\tilde{\mathbf{q}}=\left[\begin{array}{ll}\mathbf{q}_{h} & v_{w}\end{array}\right]^{T}=\left[\begin{array}{lllll}u_{h} & v_{h} & w_{h} & p_{h} & v_{w}\end{array}\right]^{T}, \mathbf{u}=\bar{v}_{w}^{\prime}(\bar{x})$, and the operator $\mathcal{M}_{a}$ in Eq. (23) is written as

$$
\mathcal{M}_{a}=\mathbf{M}_{0}+\mathbf{M}_{1} \frac{\partial}{\partial \eta}+\mathbf{M}_{2} \frac{\partial^{2}}{\partial \eta^{2}} \text {. }
$$


The matrices $\mathcal{L}_{a}, \mathbf{M}_{0}, \mathbf{M}_{1}, \mathbf{M}_{2}$, and $\mathbf{M}_{3}$ are obtained by inspection:

$$
\begin{gathered}
\mathcal{L}_{a}=\left[\begin{array}{lllll}
F^{\prime} & 0 & 0 & 0 & 0 \\
0 & F^{\prime} & 0 & 0 & 0 \\
0 & 0 & F^{\prime} & 0 & 0 \\
1 & 0 & 0 & 0 & 0 \\
0 & 0 & 0 & 0 & 1
\end{array}\right], \quad \mathbf{M}_{0}=\left[\begin{array}{cccccc}
i+\frac{\eta F^{\prime \prime}}{2 \bar{x}}-\kappa^{2} & -F^{\prime \prime} & 0 & 0 & 0 \\
\frac{\eta\left(\eta F^{\prime}\right)^{\prime}-F}{(2 \bar{x})^{2}} & i-\frac{\left(\eta F^{\prime}\right)^{\prime}}{2 \bar{x}}-\kappa^{2} & 0 & 0 & 0 \\
0 & 0 & i-\kappa^{2} & \kappa^{2} & 0 \\
0 & 0 & -1 & 0 & 0 \\
0 & 0 & 0 & 0 & 0
\end{array}\right], \\
\mathbf{M}_{1}=\left[\begin{array}{cccccc}
\frac{F}{2 \bar{x}} & 0 & 0 & 0 & 0 \\
0 & \frac{F}{2 \bar{x}} & 0 & -\frac{1}{2 \bar{x}} & 0 \\
0 & 0 & \frac{F}{2 \bar{x}} & 0 & 0 \\
\frac{\eta}{2 \bar{x}} & -1 & 0 & 0 & 0 \\
0 & 0 & 0 & 0 & 0
\end{array}\right], \quad \mathbf{M}_{2}=\left[\begin{array}{ccccc}
\frac{1}{2 \bar{x}} & 0 & 0 & 0 & 0 \\
0 & \frac{1}{2 \bar{x}} & 0 & 0 & 0 \\
0 & 0 & \frac{1}{2 \bar{x}} & 0 & 0 \\
0 & 0 & 0 & 0 & 0 \\
0 & 0 & 0 & 0 & 0
\end{array}\right], \quad \mathbf{M}_{3}=\left[\begin{array}{c}
-F^{\prime} u_{p} \\
-F^{\prime} v_{p} \\
-F^{\prime} w_{p} \\
-u_{p} \\
1
\end{array}\right] .
\end{gathered}
$$

The objective is to minimize the augmented cost function

$$
\begin{aligned}
J= & \frac{1}{2} \int_{0}^{\infty} \tilde{\mathbf{q}}\left(\bar{x}_{f}\right)^{\star} \mathbf{P}\left(\bar{x}_{f}\right) \tilde{\mathbf{q}}\left(\bar{x}_{f}\right) d \eta+\frac{1}{2} \int_{\bar{x}_{0}}^{\bar{x}_{f}} \int_{0}^{\infty}\left(\tilde{\mathbf{q}}^{\star} \mathbf{Q} \tilde{\mathbf{q}}+\mathbf{u}^{\star} \mathbf{R u}\right) d \eta d \bar{x} \\
& +\int_{\bar{x}_{0}}^{\bar{x}_{f}} \int_{0}^{\infty} \mathbf{v}^{\star}\left(\frac{\partial \mathcal{L}_{a} \tilde{\mathbf{q}}}{\partial \bar{x}}-\mathbf{M}_{0} \tilde{\mathbf{q}}-\mathbf{M}_{1} \frac{\partial \tilde{\mathbf{q}}}{\partial \eta}-\mathbf{M}_{2} \frac{\partial^{2} \tilde{\mathbf{q}}}{\partial \eta^{2}}-\mathbf{M}_{3} \mathbf{u}\right) d \eta d \bar{x} \\
& +\left.\int_{\bar{x}_{0}}^{\bar{x}_{f}} \mathbf{z}^{\star}\left(\mathbf{D}_{2} \frac{\partial \mathbf{q}_{h}}{\partial \eta}+\mathbf{D}_{1} \mathbf{q}_{h}-\mathbf{D}\right)\right|_{\eta=\infty} d \bar{x},
\end{aligned}
$$

where $\mathbf{v}=\left[\begin{array}{lll}u^{+} & v^{+} w^{+} p^{+} v_{w}^{+}\end{array}\right]^{T}$ and $\mathbf{z}=\left[\begin{array}{llll}z_{u} & z_{v} & z_{w} & z_{p}\end{array}\right]^{T}$ are the adjoint vectors. The last integral in Eq. (60) is related to the free-stream boundary conditions (12) and (13). Matrices $\mathbf{D}_{2}, \mathbf{D}_{1}$, and $\mathbf{D}$ are obtained by inspection.

The matrix $\mathbf{Q}$ should be such that $\tilde{\mathbf{q}}^{\star} \mathbf{Q} \tilde{\mathbf{q}}=f(\kappa)|\bar{u}|^{2}$, where $f(\kappa)$ is an as yet unknown function of $\kappa$. Similarly, matrix $\mathbf{P}\left(\bar{x}_{f}\right)$ should be such that $\tilde{\mathbf{q}}\left(\bar{x}_{f}\right)^{\star} \mathbf{P}\left(\bar{x}_{f}\right) \tilde{\mathbf{q}}\left(\bar{x}_{f}\right)=P\left(\bar{x}_{f}\right) f(\kappa)\left|\bar{u}\left(\bar{x}_{f}\right)\right|^{2}$, where $P\left(\bar{x}_{f}\right)$ is the penalty weight of the final state. Using (28), $\mathbf{Q}$ takes the form

$$
\mathbf{Q}=f(\kappa)\left[\begin{array}{ccccc}
1 & 0 & 0 & 0 & u_{p} \\
0 & 0 & 0 & 0 & 0 \\
0 & 0 & 0 & 0 & 0 \\
0 & 0 & 0 & 0 & 0 \\
\bar{u}_{p} & 0 & 0 & 0 & \bar{u}_{p} u_{p}
\end{array}\right]
$$

Setting the first variation of $J$ with respect to $\tilde{\mathbf{q}}$ equal to 0 results in the adjoint equations:

$$
-\mathcal{L}_{a}^{\star} \frac{\partial \mathbf{v}}{\partial \bar{x}}=\mathbf{M}_{0}^{\star} \mathbf{v}-\frac{\partial \mathbf{M}_{1}^{\star} \mathbf{v}}{\partial \eta}+\frac{\partial}{\partial \eta}\left(\frac{\partial \mathbf{M}_{2}^{\star} \mathbf{v}}{\partial \eta}\right)-\mathbf{Q q},
$$

or in expanded form

$$
\begin{aligned}
-F^{\prime} \frac{\partial u^{+}}{\partial \bar{x}}-\frac{\partial p^{+}}{\partial \bar{x}}= & \left(-i+\frac{\eta F^{\prime \prime}}{2 \bar{x}}-\kappa^{2}\right) u^{+}+\frac{1}{(2 \bar{x})^{2}}\left[\eta\left(\eta F^{\prime}\right)^{\prime}-F\right] v^{+}-\frac{1}{2 \bar{x}} \frac{\partial\left(F u^{+}\right)}{\partial \eta} \\
& -\frac{1}{2 \bar{x}} \frac{\partial\left(\eta p^{+}\right)}{\partial \eta}+\frac{1}{2 \bar{x}} \frac{\partial^{2} u^{+}}{\partial \eta^{2}}-f(\kappa) u_{h}-f(\kappa) u_{p} v_{w}
\end{aligned}
$$




$$
\begin{gathered}
-F^{\prime} \frac{\partial v^{+}}{\partial \bar{x}}=-F^{\prime \prime} u^{+}-\left[i+\frac{\left(\eta F^{\prime}\right)^{\prime}}{2 \bar{x}}+\kappa^{2}\right] v^{+}-\frac{1}{2 \bar{x}} \frac{\partial\left(F v^{+}\right)}{\partial \eta}+\frac{\partial p^{+}}{\partial \eta}+\frac{1}{2 \bar{x}} \frac{\partial^{2} v^{+}}{\partial \eta^{2}} \\
-F^{\prime} \frac{\partial w^{+}}{\partial \bar{x}}=-\left(i+\kappa^{2}\right) w^{+}-p^{+}-\frac{1}{2 \bar{x}} \frac{\partial\left(F w^{+}\right)}{\partial \eta}+\frac{1}{2 \bar{x}} \frac{\partial^{2} w^{+}}{\partial \eta^{2}} \\
\kappa^{2} w^{+}+\frac{1}{2 \bar{x}} \frac{\partial v^{+}}{\partial \eta}=0 \\
\frac{d v_{w}^{+}}{d \bar{x}}=f(\kappa) \bar{u}_{p}\left(u_{h}+u_{p} v_{w}\right) .
\end{gathered}
$$

At $\eta=0$, the boundary conditions are

$$
u^{+}=v^{+}=w^{+}=0 .
$$

As $\eta \rightarrow \infty$, the boundary conditions are

$$
\begin{aligned}
& u^{+}=v^{+}=0 \\
& p^{+}+\frac{1}{2 \bar{x}} \frac{\partial v^{+}}{\partial \eta}=0 \\
& \frac{\partial w^{+}}{\partial \eta}+\left[|\kappa|(2 \bar{x})^{1 / 2}-F\right] w^{+}=0 .
\end{aligned}
$$

As $F \rightarrow \bar{\eta}=\eta-\beta$ with $\beta=1.217$ as $\eta \rightarrow \infty$, expression (69) becomes

$$
\frac{\partial w^{+}}{\partial \eta}+\left[|\kappa|(2 \bar{x})^{1 / 2}-\bar{\eta}\right] w^{+}=0
$$

Boundary conditions (69) and (70) differ from those of Ref. [12] because in their case the boundary conditions as $\eta \rightarrow \infty$ are $u=w=p=0$, as opposed to (12) and (13) in our case.

The solution of the adjoint system is now examined. We first recall that the LUBR system (20) for the particular solution $\mathbf{q}_{p}$ [or in expanded form (7)-(10)] admits an asymptotic self-similar solution in the limit of $\kappa \rightarrow \infty$ of the form [23]

$$
\left\{u_{p}, v_{p}, w_{p}, p_{p}\right\}=\left\{\kappa^{-2} \hat{u}_{p}, \hat{v}_{p}, \hat{w}_{p}, \hat{p}_{p}\right\}\left(\kappa^{2} \bar{x}, \eta\right) .
$$

In limit $\kappa \rightarrow \infty$, the unsteady terms in the three momentum equations vanish, so the velocity and pressure in Eq. (71) satisfy the steady momentum equations exactly. In practice, solutions with $\kappa$ larger than about 1 approach the asymptotic solutions well. Note that the particular solution $\mathbf{q}_{p}$ depends only on $\kappa$, and not on $\kappa_{2}$, as the latter parameter enters only through the outer boundary condition, which is imposed only on $\mathbf{q}_{h}$.

Inspection of the direct system (56) shows that it admits the asymptotic solution

$$
\left\{u_{h}, v_{h}, w_{h}, p_{h}\right\}=\left\{\kappa^{-2} \hat{u}_{h}, \hat{v}_{h}, \hat{w}_{h}, \hat{p}_{h}\right\}\left(\kappa^{2} \bar{x}, \eta ; \frac{\kappa_{2}}{\kappa}\right)
$$

if $\bar{v}_{w}^{\prime}(\bar{x})$ scales as

$$
\frac{d \bar{v}_{w}}{d \bar{x}}=\kappa^{2} \frac{d \hat{\bar{v}}_{w}}{d \hat{\bar{x}}} \quad \text { with } \quad \hat{\bar{x}}=\kappa^{2} \bar{x}
$$

or, equivalently, $v_{w}=\hat{v}_{w}\left(\kappa^{2} \bar{x}, \eta ; \kappa_{2} / \kappa\right)$. If (73) is satisfied, then the controlled field $\overline{\mathbf{q}}=\mathbf{q}_{h}+$ $\bar{v}_{w}(\bar{x}) \mathbf{q}_{p}$ will also satisfy the same scalings:

$$
\{\bar{u}, \bar{v}, \bar{w}, \bar{p}\}=\left\{\kappa^{-2} \hat{u}, \hat{v}, \hat{w}, \hat{p}\right\}\left(\kappa^{2} \bar{x}, \eta ; \frac{\kappa_{2}}{\kappa}\right) .
$$

Using these scalings for the direct system and taking $f(\kappa)=\kappa^{2}$ to compensate for the scaling of $u_{p}$ [refer to the first of (71)], it is found that the adjoint system admits the following self-similar 
solution as $\kappa \rightarrow \infty$ :

$$
\left\{u^{+}, v^{+}, w^{+}, p^{+}, v_{w}^{+}\right\}=\left\{\kappa^{-2} \hat{u}^{+}, \kappa^{-4} \hat{v}^{+}, \kappa^{-4} \hat{w}^{+}, \kappa^{-2} \hat{p}^{+}, \kappa^{-4} \hat{v}_{w}^{+}\right\}\left(\kappa^{2} \bar{x}, \eta ; \frac{\kappa_{2}}{\kappa}\right) .
$$

It is worth noticing that these scalings are different from the scalings (71) of the LUBR equations. The conditions under which (73) is satisfied need to be established. Setting the first variation of $J$ with respect to $\mathbf{u}$ equal to 0 results in the optimality condition:

$$
\frac{d \bar{v}_{w}}{d \bar{x}}=\mathbf{u}=\mathbf{R}^{-1} \mathbf{M}_{3}^{\star} \mathbf{v}
$$

and, assuming that $\mathbf{R}$ is a diagonal matrix, for example, $\mathbf{R}=r^{2} \mathbf{I}$,

$$
\frac{d \bar{v}_{w}}{d \bar{x}}=\frac{1}{r^{2}} \mathbf{M}_{3}^{\star} \mathbf{v}=\frac{1}{r^{2}}\left[-F^{\prime} \bar{u}_{p}-F^{\prime} \bar{v}_{p}-F^{\prime} \bar{w}_{p}-\bar{u}_{p} 1\right]\left[\begin{array}{c}
u^{+} \\
v^{+} \\
w^{+} \\
p^{+} \\
v_{w}^{+}
\end{array}\right],
$$

where the overbar here denotes complex conjugate. Substitution of (71) and (75) into (77) yields

$$
\frac{d \bar{v}_{w}}{d \bar{x}}=O\left(r^{-2} \kappa^{-4}\right)
$$

and, in order to obtain (73), $r^{-2} \kappa^{-4}=O\left(\kappa^{2}\right) \Rightarrow r^{2}=O\left(\kappa^{-6}\right)$, so the weighting matrix $\mathbf{R}$ must have the form $\mathbf{R}=a^{2} \kappa^{-6} \mathbf{I}$ (where $a^{2}$ is a constant parameter).

We will now derive the scaling for the minimum value of the cost function, $J_{\min }$. Ignoring penalization of the final state we have

$$
J_{\min }\left(\kappa, \kappa_{2}\right)=\frac{1}{2} \int_{\bar{x}_{0}}^{\bar{x}_{f}} \int_{0}^{\infty}\left(\kappa^{2}|u|^{2}+\frac{a^{2}}{\kappa^{6}}\left|\frac{d v_{w}}{d \bar{x}}\right|^{2}\right) d \eta d \bar{x} .
$$

We have shown that $u=\kappa^{-2} \hat{u}\left(\kappa^{2} \bar{x}, \eta ; \kappa_{2} / \kappa\right), \frac{d v_{w}}{d \bar{x}}=\kappa^{2} \frac{d \hat{v}_{w}}{d \hat{x}}$, and $\bar{x}=\hat{\bar{x}} \kappa^{-2}$, and substituting into (79) one finds

$$
J_{\min }\left(\kappa, \kappa_{2}\right)=\frac{1}{2} \int_{\bar{x}_{0}}^{\bar{x}_{f}} \int_{0}^{\infty}\left(\frac{1}{\kappa^{4}}|\hat{u}|^{2}+\frac{a^{2}}{\kappa^{4}}\left|\frac{d \hat{v}_{w}}{d \hat{\bar{x}}}\right|^{2}\right) d \eta d \hat{\bar{x}}
$$

or

$$
J_{\min }\left(\kappa, \kappa_{2}\right)=\frac{1}{\kappa^{4}} \hat{J}\left(\kappa, \kappa_{2}\right)
$$

where

$$
\hat{J}_{\min }\left(\kappa, \kappa_{2}\right)=\frac{1}{2} \int_{\bar{x}_{0}}^{\bar{x}_{f}} \int_{0}^{\infty}\left(|\hat{u}|^{2}+a^{2}\left|\frac{d \hat{v}_{w}}{d \hat{\bar{x}}}\right|^{2}\right) d \eta d \hat{\bar{x}} .
$$

In the next section, it will be shown that the numerical calculations confirm the above scalings.

\section{NUMERICAL RESULTS}

This section presents the results on the effect of the wall-based controller on the individual modes and on the full-spectrum streaks. When applied to the full spectrum, the results will be compared against experimental data without control. The weighting matrix $\mathbf{R}_{i}$ was set to

$$
\mathbf{R}_{i}=\left\{\begin{array}{cc}
a^{2} \kappa^{-6} \mathbf{I} & \text { if } \kappa>1 \\
a^{2} \mathbf{I} & \text { if } \kappa \leqslant 1
\end{array}\right.
$$



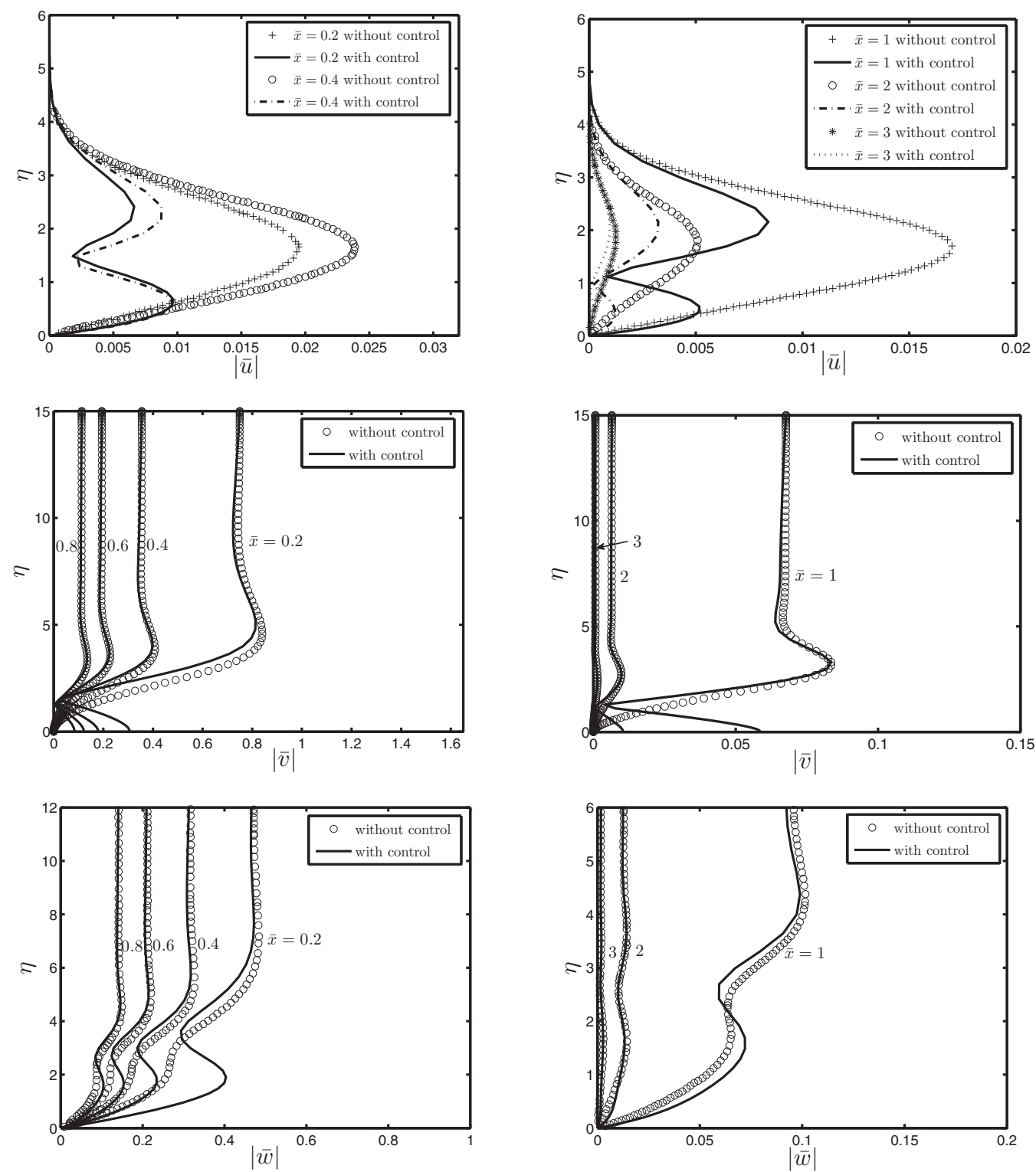

FIG. 3. Profiles of the (top) streamwise, (middle) wall-normal, and (bottom) spanwise perturbation velocity magnitudes at various $\bar{x}$ locations without and with control (for $\kappa=1, \kappa_{2}=-1, a^{2}=10^{-6}$ ).

The matrix $\mathbf{R}_{i}$ was set equal to the constant $a^{2} \mathbf{I}$ in order to avoid excessive penalization of the control signal when $\kappa \leqslant 1$. It was found unnecessary to penalize the final state, so the matrix $\mathbf{P}_{N}$ was set to 0. The numerical code was thoroughly validated against the uncontrolled reference data in Ref. [23] (see Appendix B).

\section{A. Control of individual modes}

Figure 3 shows profiles of the amplitudes of the streamwise, wall-normal, and spanwise perturbation velocities with and without control at the wall surface. The amplitude of the streamwise velocity is significantly reduced to less than half compared with the uncontrolled case. It is interesting 

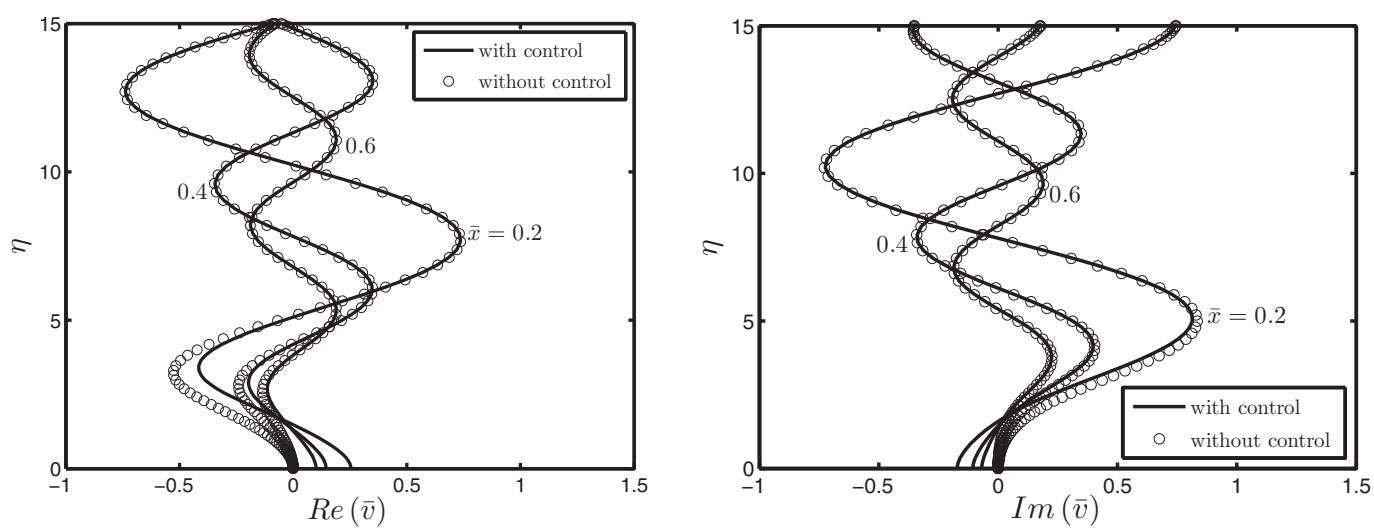

FIG. 4. Profiles of the real (left) and imaginary (right) parts of the wall-normal perturbation velocity $\bar{v}$ at various $\bar{x}$ locations without and with control (for $\kappa=1, \kappa_{2}=-1, a^{2}=10^{-6}$ ).

to note that the single peak in the middle of the boundary layer disappears and two peaks occur, one close to the wall, at about $\eta=0.5$, and the other farther away from the wall, slightly above $\eta=2$. The appearance of the near-wall peak is due to the wall-normal control velocity $\bar{v}_{w}$. Through continuity, Eq. (10), the wall-normal gradient of the wall-normal transpiration velocity induces a streamwise-varying streamwise velocity near the wall.

The magnitudes of the wall-normal and spanwise components of the perturbation velocity at various $\bar{x}$ locations are also shown in Fig. 3. The magnitude of the wall-normal velocity is large very near the wall because of the wall transpiration, and it reduces to a minimum at about $\eta=1.5$ from the wall. Beyond this minimum, the profile quickly recovers the amplitude in the uncontrolled case. Near the wall and especially in the upstream region in the proximity of the leading edge, the magnitude of the spanwise velocity is larger than in the uncontrolled case. Similarly to the creation of the near-wall peak in the streamwise-velocity profile, the augmented spanwise velocity is caused by continuity to balance the intense wall-normal gradient of the wall-transpiration velocity. As the intensity of the wall transpiration decreases downstream, the near-wall spanwise velocity eventually matches the uncontrolled values, whereas the effect on the near-wall streamwise velocity is more persistent and differs substantially from the uncontrolled value even up to $\bar{x}=3$ where the wall transpiration is null.

As for the wall-normal velocity, the control signal does not affect the spanwise velocity away from the wall. This proves that the area significantly affected by the wall transpiration is confined near the wall, and the development of the boundary-layer perturbation far away from the wall is still dominated by the excitation of free-stream turbulence, as expected.

The previous figures show only the magnitudes of velocities but do not convey all the information necessary to examine the action of the controller in more detail. For this, it is instructive to look at the real and imaginary parts of the $\bar{v}$ velocity; these are shown in Fig. 4. Away from the wall (for $\eta \geqslant 5$ ) the curves for the real and imaginary parts with and without control collapse. The controller modifies the near-wall field by introducing an actuation velocity of opposite sign to the wall-normal velocity farther away. This is achieved very smoothly. For example, for the real part at $\bar{x}=0.2 \mathrm{a}$ small deviation of the profile to the right leads to a (positive) wall-normal velocity at the wall that opposes the negative velocities farther away, leading to a stagnation point at $\eta \approx 2$. The behavior is qualitatively similar for the imaginary parts (right panel). The fact that both real and imaginary parts change sign across the stagnation point leads to a $180^{\circ}$ phase jump for the controlled case (figure not shown for brevity).

To obtain a clearer picture of the development of the perturbation velocities, Fig. 5 presents contour plots of the amplitude of the streamwise perturbation velocity, $|\bar{u}|$, without control and for three different values of the control weight parameter $a^{2}$. The actuation velocity creates a buffer 


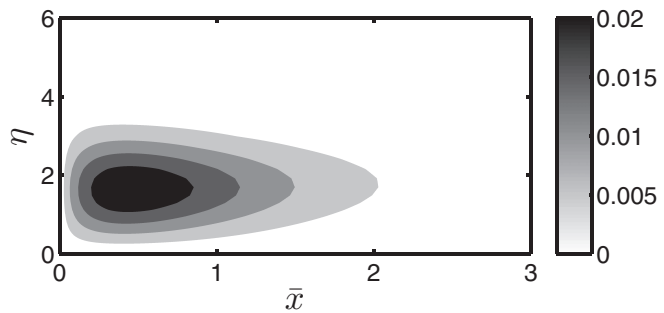

(a)

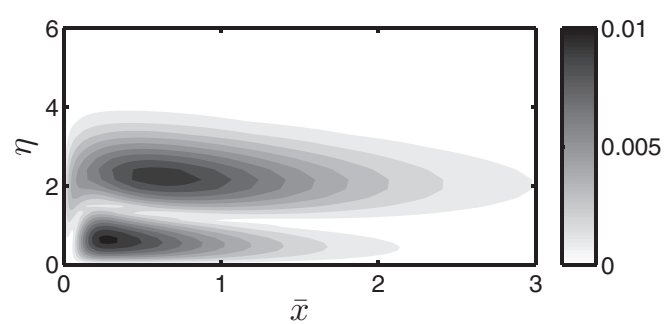

(c)

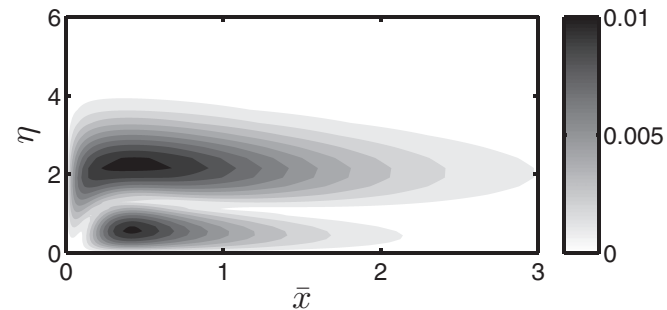

(b)

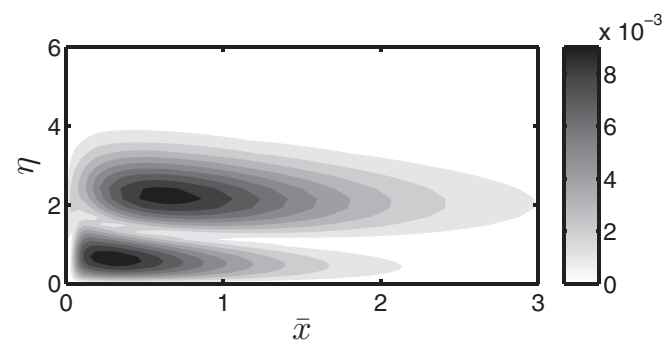

(d)

FIG. 5. Contour plot of the amplitude of the streamwise perturbation velocity, $|\bar{u}|$ (a) without control and with control using (b) $a^{2}=10^{-4}$, (c) $a^{2}=10^{-5}$, (d) $a^{2}=10^{-6}$ (for $\kappa=1, \kappa_{2}=-1$ ).

vortex between the wall and the main vortex, which results in the latter being lifted to larger $\eta$ values. The presence of this buffer vortex explains the two peaks in Fig. 3.

When the value of $a^{2}$ is reduced, that is, for cheaper wall actuation, the wall-transpiration velocity achieves higher values closer to the leading edge (as shown in the right part of Fig. 6) and the buffer vortex moves upstream displacing the main vortex downstream. The wall-normal locations of the two vortices is largely unchanged as $a^{2}$ varies. Similar buffer vortices have been observed in a channel flow by Bewley and Liu [7].

Figure 6 shows the effect of $a^{2}$ on the downstream evolution of $\kappa^{2}|\bar{u}|$ at $\eta=2$ and the magnitude of the wall-transpiration velocity $\left|\bar{v}_{w}\right|$. The effect of the control weight becomes important for values of $\kappa^{2} \bar{x}$ smaller than about 0.9 . For higher values all curves collapse to a single profile. The peak of $\kappa^{2}|\bar{u}|$ is slightly reduced as $a^{2}$ decreases from $10^{-4}$ to $10^{-5}$, but the location moves downstream
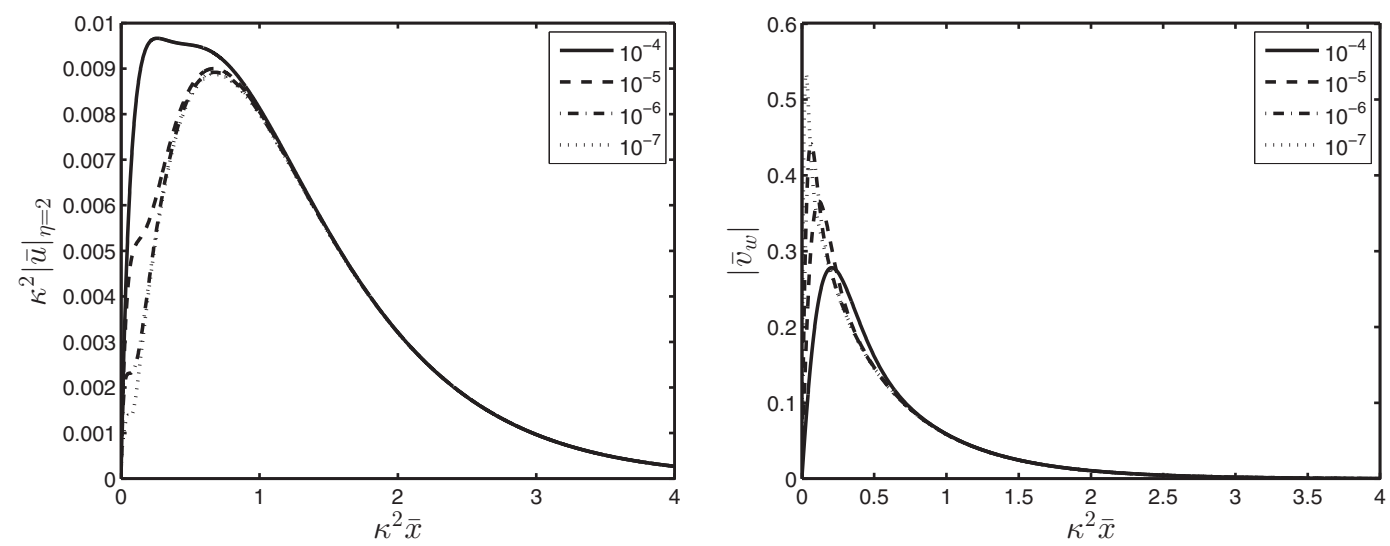

FIG. 6. Variation of $\kappa^{2}|\bar{u}|$ (left) and $\left|\bar{v}_{w}\right|$ (right) along $\kappa^{2} \bar{x}$ at $\eta=2$ for $\kappa=1, \kappa_{2}=-1$, and different values of $a^{2}$. 

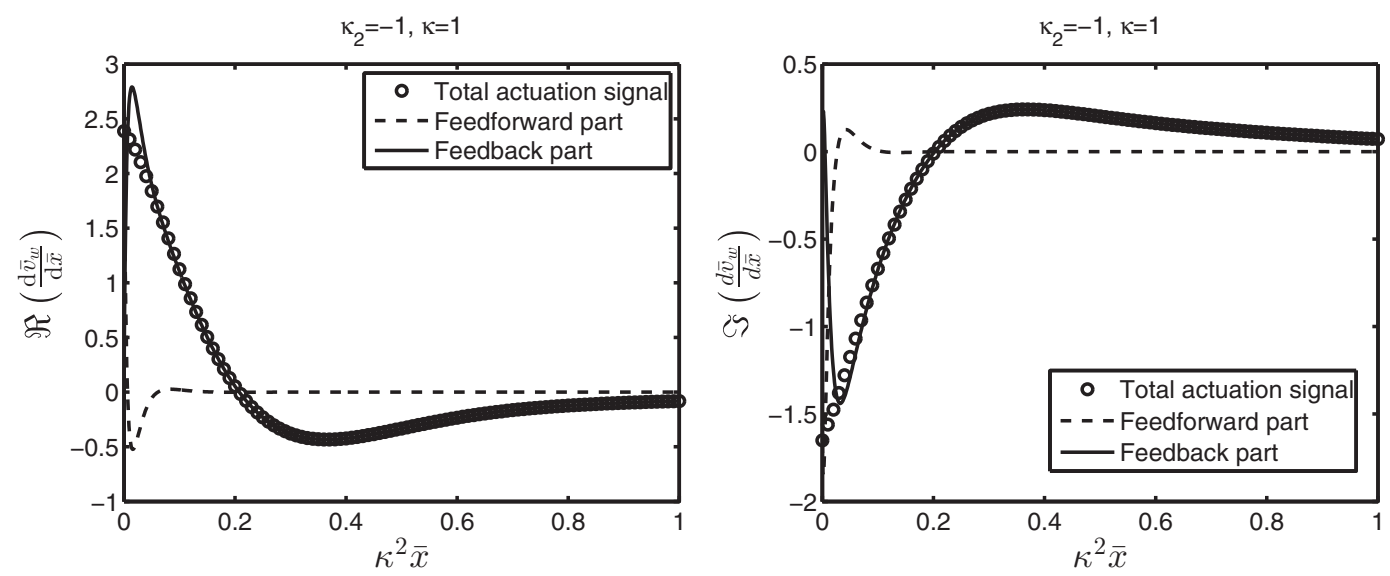

FIG. 7. Variation of the real (left) and imaginary (right) parts of the control signal along $\kappa^{2}|\bar{x}|$ for $\kappa_{2}=$ $-1, \kappa=-1, a^{2}=10^{-4}$. The feedback, feed-forward, and total signal are shown separately.

(due to the displacement of the main vortex by the buffer vortex shown in Fig. 5). For values of $a^{2}$ smaller than $10^{-5}$ the peak remains at the same location and the effect of the reduction of $a^{2}$ is confined in the upstream region, that is, between the leading edge and the peak location. In this region, the magnitude of the actuation velocity increases (as shown in the right part of the figure) leading to strong suppression of $\kappa^{2}|\bar{u}|$. The peak of the actuation velocity is located upstream of the peak of $\kappa^{2}|\bar{u}|$. It is also worth noticing that by reducing $a^{2}$, the velocity profiles collapse to a single curve over a larger range of $\kappa^{2} \bar{x}$ values. This curve represents the minimum values of $\kappa^{2}|\bar{u}|$ that can achieved using the present control approach.

It was shown in Sec. $\mathrm{V}$ that the control signal consists of two parts, a feedback part and a feed-forward part. The real and imaginary parts of the control signal (streamwise derivative of wall actuation) are shown in Fig. 7. The feed-forward part is acting only very close to the leading edge, and it reduces to very small values for $\bar{x} \geqslant 0.1$. Therefore, it is expected that its contribution to the minimum value of the cost function $J_{\text {min }}$ will be very small.

In order to examine this further, we consider the analytic expression for $J_{\min }$ derived in Appendix D:

$$
J_{\min }=\frac{1}{2} \mathbf{q}_{0}^{\star} \mathbf{P}_{0} \mathbf{q}_{0}-\frac{1}{2}\left(\mathbf{V}_{0}^{\star} \mathbf{q}_{0}+\mathbf{q}_{0}^{\star} \mathbf{V}_{0}\right)+\frac{1}{2} \sum_{i=0}^{N-1} \mathbf{W}_{i},
$$

where the sequence $\mathbf{W}_{i}$ is computed from the following expression by marching backwards:

$$
\begin{aligned}
\mathbf{W}_{i}= & -\left(\mathbf{V}_{i+1}-\mathbf{P}_{i+1} \mathbf{C}_{i}\right)^{\star} \mathbf{B}_{i}\left[\left(\mathbf{B}_{i}^{\star} \mathbf{P}_{i+1} \mathbf{B}_{i}+\mathbf{R}_{i}\right)^{\star}\right]^{-1} \mathbf{B}_{i}^{\star}\left(\mathbf{V}_{i+1}-\mathbf{P}_{i+1} \mathbf{C}_{i}\right) \\
& -\left(\mathbf{C}_{i}^{\star} \mathbf{V}_{i+1}+\mathbf{V}_{i+1}^{\star} \mathbf{C}_{i}\right)+\mathbf{C}_{i}^{\star} \mathbf{P}_{i+1} \mathbf{C}_{i} .
\end{aligned}
$$

We checked the validity of this expression by computing $J_{\min }$ with direct numerical integration of (41) and from (84); the results are identical, as shown in the left part of Fig. 8 for different values of $\kappa$ and constant ratio $\kappa_{2} / \kappa=-1$.

We can rearrange the analytic expression for $J_{\min }$ and write it as the sum of two components that correspond to the feedback and feed-forward parts of the control signal as follows:

$$
J_{\min }=J_{f b}-J_{f f},
$$



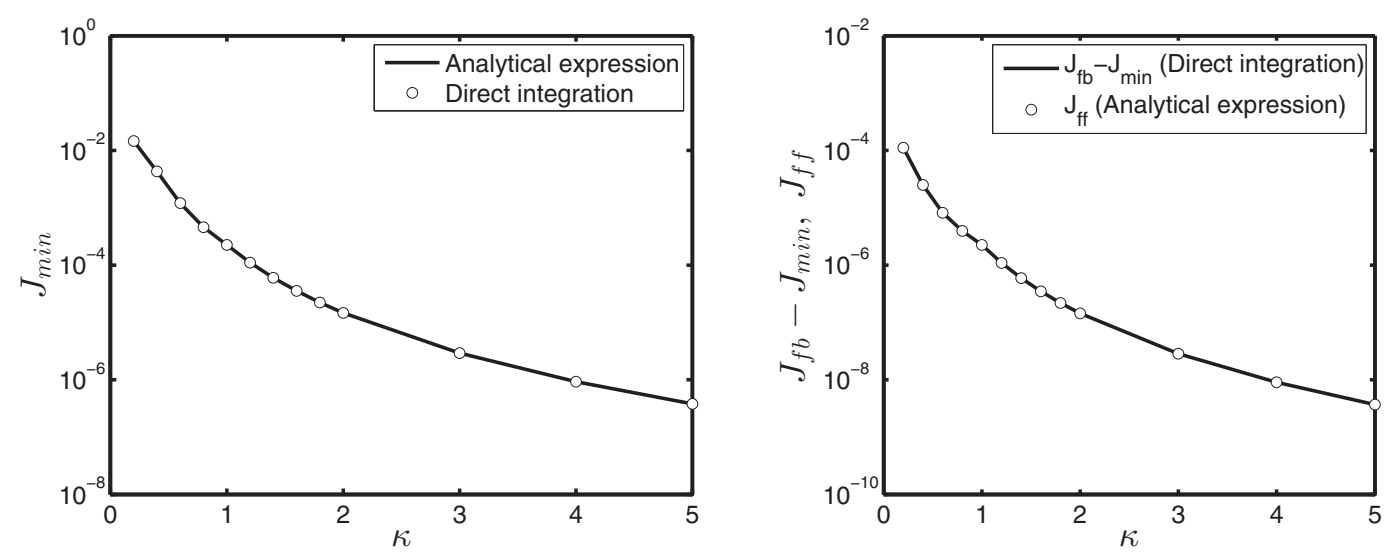

FIG. 8. Comparison between $J_{\min }$ obtained using direct numerical integration and the closed form analytical expression (84) (left) and between $J_{f b}-J_{\min }$ computed numerically and $J_{f f}$ computed analytically (right). For all cases $\kappa_{2} / \kappa=-1$.

where

$$
\begin{gathered}
J_{f b}=\frac{1}{2} \mathbf{q}_{0}^{\star} \mathbf{P}_{0} \mathbf{q}_{0}-\frac{1}{2}\left(\mathbf{V}_{0}^{\star} \mathbf{q}_{0}+\mathbf{q}_{0}^{\star} \mathbf{V}_{0}\right)+\frac{1}{2} \sum_{i=0}^{N-1}\left[-\left(\mathbf{C}_{i}^{\star} \mathbf{V}_{i+1}+\mathbf{V}_{i+1}^{\star} \mathbf{C}_{i}\right)+\mathbf{C}_{i}^{\star} \mathbf{P}_{i+1} \mathbf{C}_{i}\right], \\
J_{f f}=\frac{1}{2} \sum_{i=0}^{N-1}\left(\mathbf{V}_{i+1}-\mathbf{P}_{i+1} \mathbf{C}_{i}\right)^{\star} \mathbf{B}_{i}\left[\left(\mathbf{B}_{i}^{\star} \mathbf{P}_{i+1} \mathbf{B}_{i}+\mathbf{R}_{i}\right)^{\star}\right]^{-1} \mathbf{B}_{i}^{\star}\left(\mathbf{V}_{i+1}-\mathbf{P}_{i+1} \mathbf{C}_{i}\right) .
\end{gathered}
$$

Physically, $J_{f b}$ is the value of cost function when only the feedback part of the control signal is applied, and the difference $J_{f b}-J_{\min }$ quantifies the effect of the feed-forward part. To confirm that this is the case, for each value of $\kappa$ we performed two simulations: one using the full control signal as given by (51) and one using only the feedback part (only the first term on the right-hand side). For each simulation, we computed the cost function using direct numerical integration of the controlled flow field, and we subtracted the results. We plot this difference and $J_{f f}$ computed from the analytic expression (88) in the right part of Fig. 8. There is excellent agreement between the numerical and analytical expressions. This confirms that $J_{f f}$ is the amount by which the cost function is reduced if the feed-forward part is added to the control signal.

It can be also noticed that the values of $J_{f f}$ are very small compared to $J_{\min }$ which demonstrates that the effect of the feed-forward component is indeed very small. This is an important result because it shows that using only the feedback part, the minimum value of the cost function $J_{f b}$ is very close to the mathematical minimum $J_{\min }$. In a practical setting one can estimate the flow field from available wall pressure and shear stress measurements and compute the feedback part. The feed-forward part appears because of the forcing in the top of the boundary layer and does not depend on the state variables. It cannot be estimated using wall measurements, but fortunately its effect is very small.

In Sec. VI it was shown that the controlled flow, for appropriate weighting matrices, has selfsimilar behavior. Figure 9 shows the variation of $\kappa^{2}|\bar{u}|$ at $\eta=2$ and of the control velocity $\left|v_{w}\right|$ along $\kappa^{2}|\bar{x}|$ for different values of $\kappa$ and clearly demonstrates that the numerically computed controlled flow indeed satisfies the analytically derived self-similarity.

It was also shown that the adjoint variables have different scalings compared to velocity and pressure. Figure 10 confirms these scalings of the adjoint variables $u^{+}, v^{+}$numerically. The profiles of $w^{+}$and $p^{+}$(not shown) also satisfy the derived similarity scalings. We also plot the scaled cost 

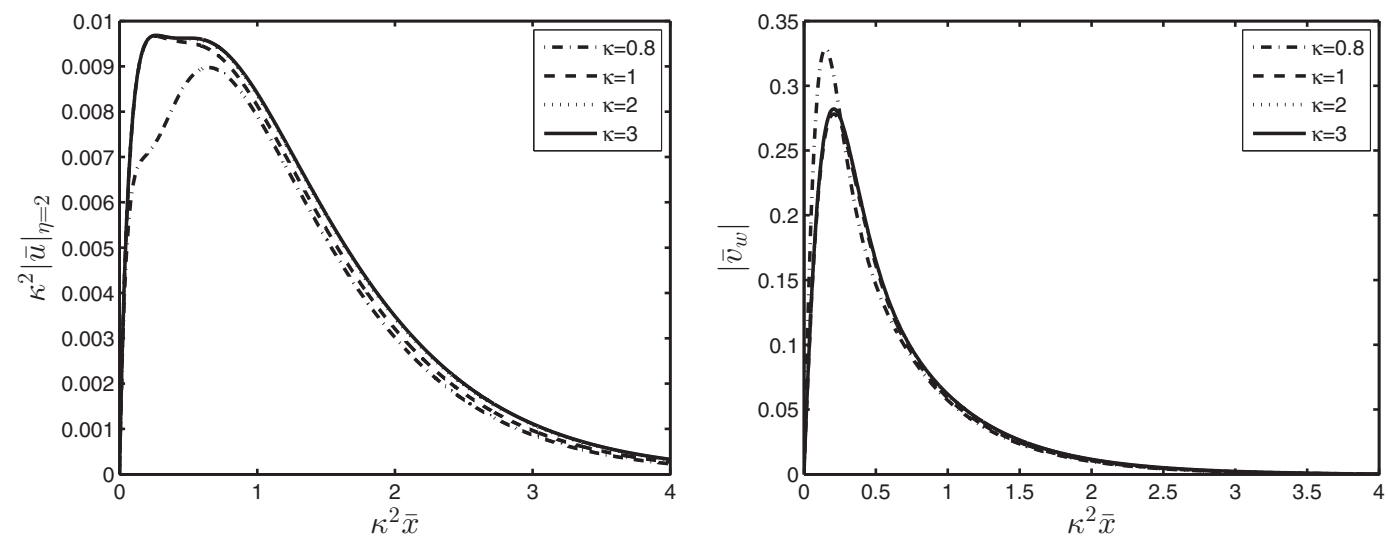

FIG. 9. Variation of $\kappa^{2}|\bar{u}|$ (left) and $\left|\bar{v}_{w}\right|$ (right) along $\kappa^{2}|\bar{x}|$ for $\kappa_{2} / \kappa=-1, a^{2}=10^{-4}$ and different values of $\kappa$.
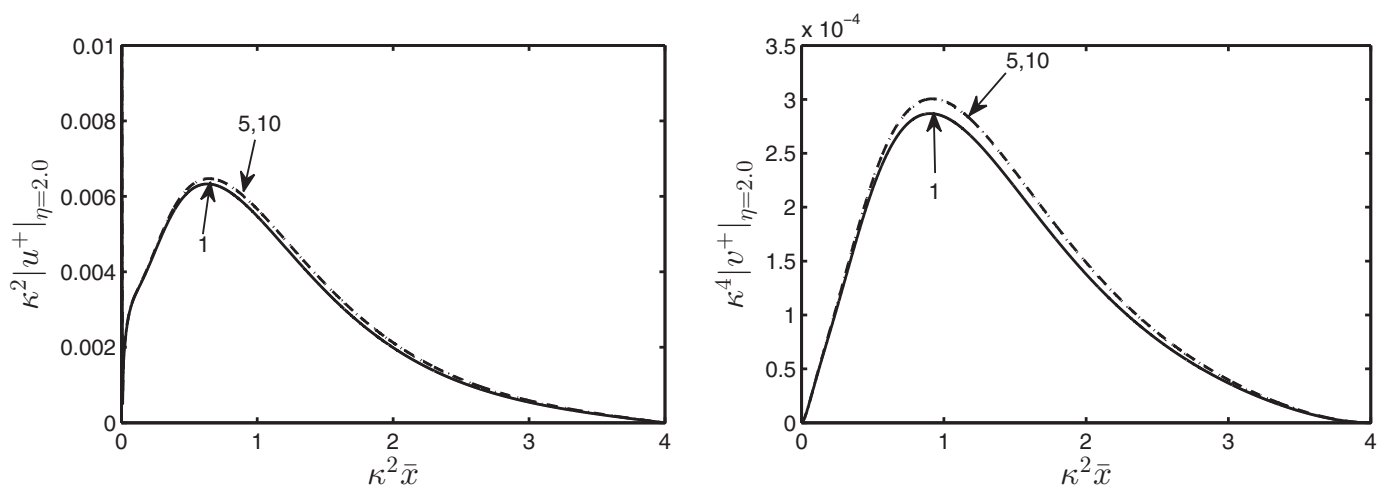

FIG. 10. Variation of $\kappa^{2}\left|u^{+}\right|$(left) and $\kappa^{4}\left|v^{+}\right|$(right) along $\kappa^{2} \bar{x}$ for $\kappa_{2} / \kappa=-1, a^{2}=10^{-4}$ and different values of $\kappa$.

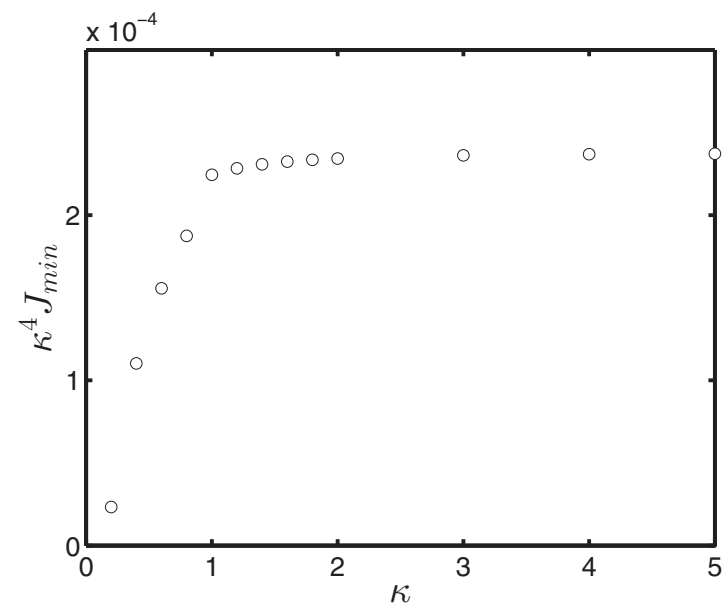

FIG. 11. Asymptotic behavior of $\kappa^{4} J_{\min }\left(\kappa_{2} / \kappa=-1\right.$ and $\left.a^{2}=10^{-4}\right)$. 


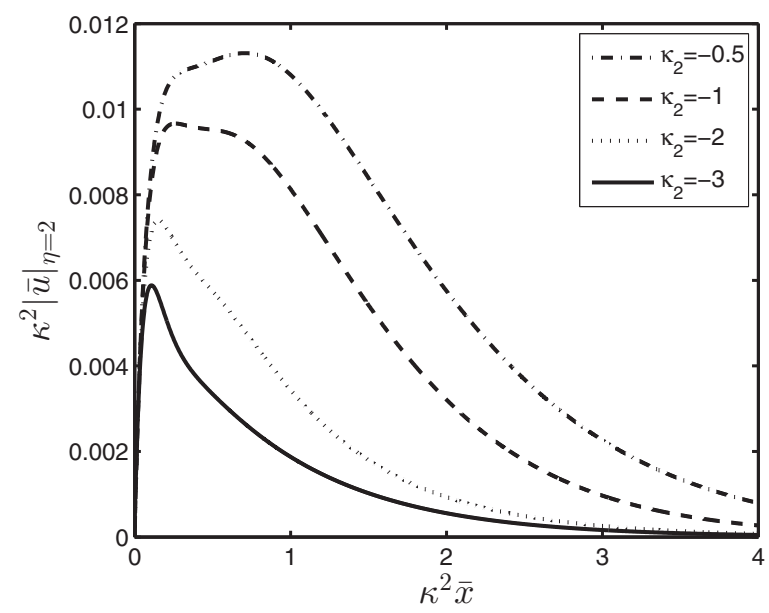

FIG. 12. Variation of $\kappa^{2}|\bar{u}|$ against $\kappa^{2} \bar{x}$ for constant $\kappa=1$ and different values of $\kappa_{2}$.

function $\kappa^{4} J_{\min }$ in Fig. 11 to demonstrate its asymptotic behavior as predicted by Eq. (81). Note that this is a replotting of the left part of Fig. 8.

We also examine the effect of the scaled wave number in the wall-normal direction $\kappa_{2}$ when the spanwise wave number $\kappa$ is kept constant. The parameter $\kappa_{2}$ does not appear in the set of evolution equations (7)-(10), and its effect enters into the system through the forcing term of Eq. (13) and the initial conditions (the effect on the latter is weak).

Figure 12 shows the effect of $\kappa_{2}$ for the same value of $\kappa=1$. For these values of $\kappa$ and $\kappa_{2}$, the initial conditions are almost indistinguishable (not shown for brevity), and therefore $\kappa_{2}$ enters only through the forcing condition at the top of the boundary layer. Increasing the value of $\kappa_{2}$ brings the peak of the excitation term closer to the leading edge and increases the decay rate. This explains why the peak of $|\bar{u}|$ moves to the left and its value is attenuated as $\kappa_{2}$ increases.

As explained in Sec. II the forcing term injects streamwise vorticity at the top of the boundary layer. Contour plots of $\bar{\omega}_{x} /\left(R_{\Lambda} k_{1}\right)^{1 / 2}$ are shown in Fig. 13 for $\kappa_{2}=-1,-2$. This plot demonstrates the penetration of the injected streamwise vortical fluctuations inside the boundary layer. Note that close to the wall the action of the controller is to generate streamwise vorticity with opposite sign to the one penetrating and therefore counteract its effect, as also mentioned before.

We close this section with a comment on the attenuation of small-amplitude Klebanoff modes, whose dynamics is described by linearized equations of motions. Although a laminar boundary layer can abruptly transition to turbulence through the secondary instability of nonlinearly saturated streaks $[39,40]$, small-amplitude streaks, such as the ones studied herein, can be a primary cause of breakdown to turbulence. It is therefore important to attenuate the amplitude of streaks described by linearized dynamics. Furthermore, linearized boundary-layer perturbations require less actuation energy than nonlinear streaks because of their smaller intensity.

Among the mechanisms through which linearized streaks can engender transition, one can recall the interaction of large-spanwise-wavelength streaks interacting with localized roughness [41] or small-wavelength Klebanoff modes encountering a localized wall perturbation or wall suction [30]. In these cases, Tollmien-Schlichting waves are triggered by the interaction between free-stream-induced streaks and the wall perturbation. These waves grow exponentially downstream and cause the breakdown of the laminar flow.

The crucial point is that the amplitude of these unstable waves, computed through receptivity analysis, is linearly proportional to the streak amplitude. The location of transition in turn depends on the amplitude of the waves. It is therefore clear that wall-based feedback control such as the one investigated herein can be effective in delaying the occurrence of transition because it attenuates the 

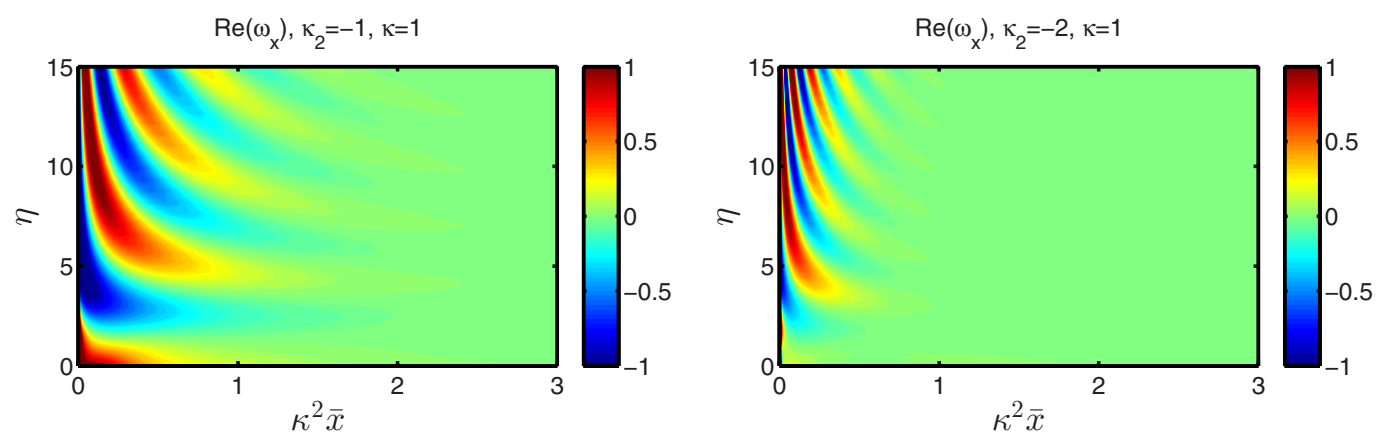

FIG. 13. Contour plots of the real part of scaled streamwise vorticity $\bar{\omega}_{x} /\left(R_{\Lambda} k_{1}\right)^{1 / 2}$ for $\kappa_{2}=-1$ (left) and $\kappa_{2}=-2$ (right).

amplitude of the streaks and of the unstable waves. Other works that prove that linearized Klebanoff modes induced by free-stream vorticity are the cause of the flow breakdown to turbulence include Refs. [42,43].

\section{B. Control of the full spectrum}

It was mentioned in Sec. IV that the formulation of the control problem makes sense only when solved for all wave numbers because it is only then that the resulting cost function computed by integration in wave number space has direct physical meaning. Therefore results from the control of the full spectrum are presented in this section.

Figure 14 shows the kernel function, defined by (35), for controlled and uncontrolled flows, $a^{2}=10^{-4}$ and $\eta=2$. This wall-normal location is higher than that used for the uncontrolled case in Ref. [23] $(\eta=1.69)$ and reflects the lifting of the primary vortex away from the wall, as shown in Fig. 5. A significant reduction in the kernel values is observed; the peak value is reduced by more than four times.

Control was then applied to a wind-tunnel flow studied experimentally by Kendall. Details about this case can be found in Ref. [23]. The free-stream velocity is $11.6 \mathrm{~ms}^{-1}$ and the turbulence level $0.26 \%$. The transverse integral length scale close to the leading edge is estimated to be $\Lambda^{*}=9 \mathrm{~mm}$. Measurements were taken at streamwise locations $\epsilon x^{*} / \Lambda^{*}=0.05-0.32$. The transverse turbulence

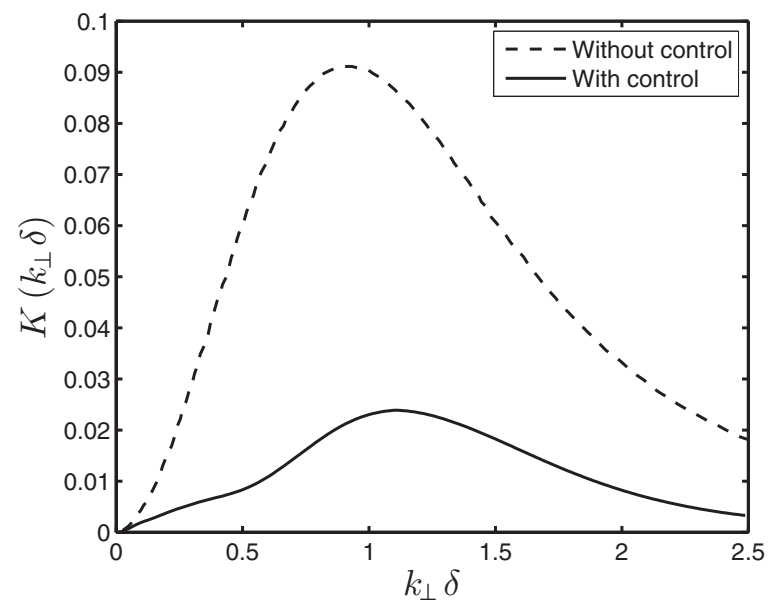

FIG. 14. Variation of the kernel function $K$ against $k_{\perp} \bar{\delta}$ for controlled $\left(a^{2}=10^{-4}\right)$ and uncontrolled flow. 


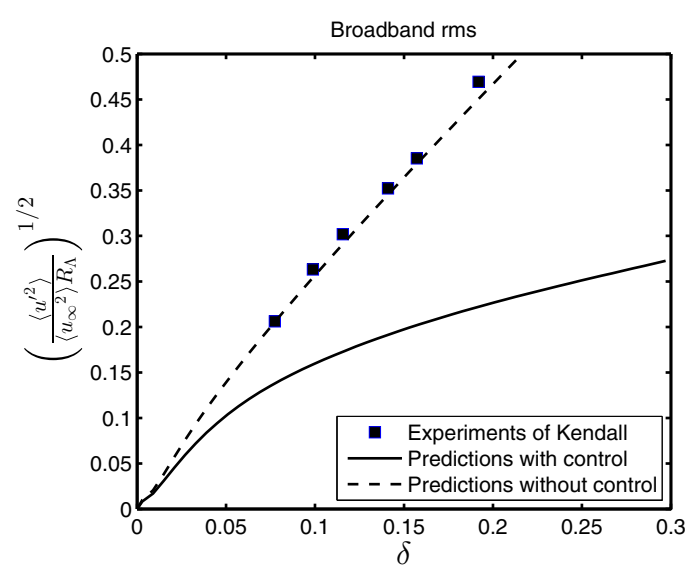

(a)

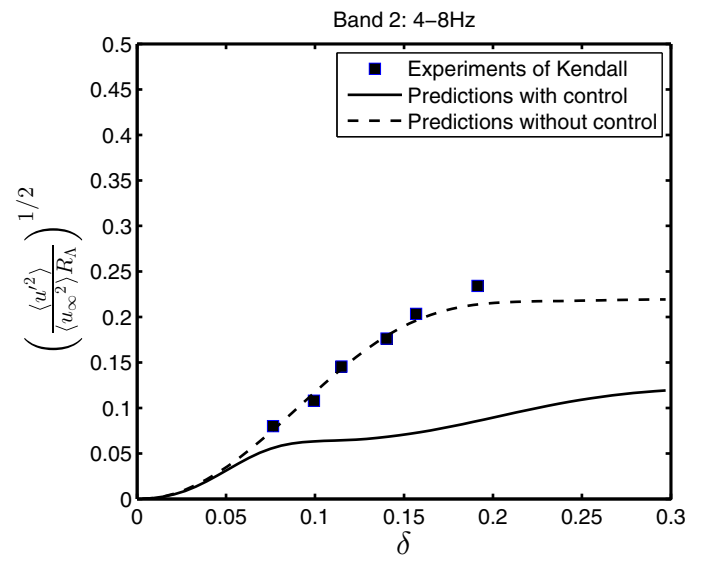

(c)

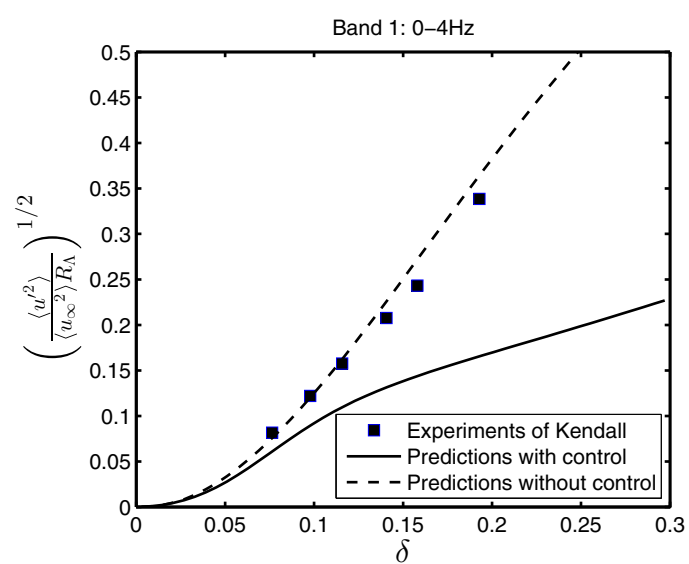

(b)

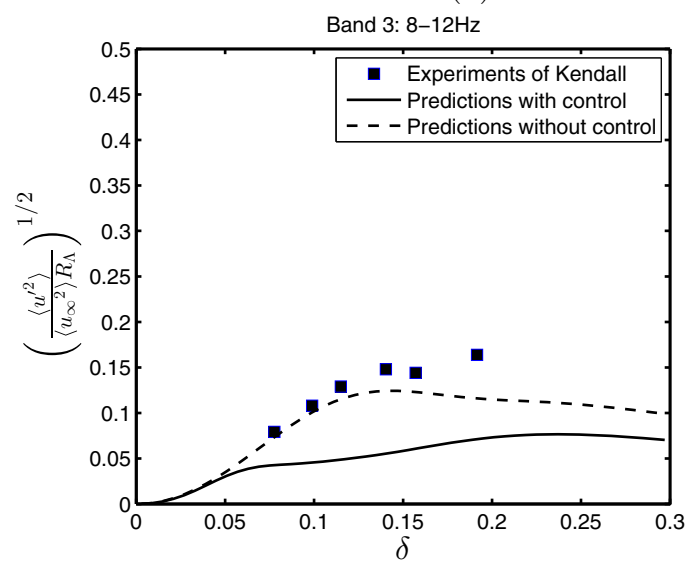

(d)

FIG. 15. Comparison of theoretical results (with and without control) with the experimental data of Kendall (a) broadband rms; (b) band 1, 0-4 Hz; (c) band 2, 4-8 Hz; and (d) band 3, 8-12 Hz.

spectrum is taken to be

$$
\Phi_{t}\left(0, k_{\perp}\right)=C \frac{\left\langle u_{\infty}^{2}\right\rangle}{\pi^{2}} k_{\perp}^{2} e^{-\left(k_{\perp}-\bar{k}_{\perp}\right)^{2} / \Delta} .
$$

Constants $\bar{k}_{\perp}=-7.0$ and $\Delta=4$ have the same values as in Ref. [23]. The process to compute constant $C$ is also explained there.

Figure 15(a) shows comparison between the values of $\left\langle u^{\prime 2}\right\rangle$ computed via (34) with and without Kendall's control and experimental data. There is good match between the experimental results and the numerical data for the open loop system. When control is applied, the initial growth is the same as this is determined by the initial conditions, but soon the effect of control becomes evident. Note the smooth variation close to the leading edge, where no overshoots appear due to the effect of the control action.

Due to linearity, the separate contributions of different frequency bands on the root mean square (rms) of velocity can be computed. The computations are now slightly more complicated because (34) and (35) are no longer decoupled. The variable $s$ on (35) is restricted between $\left[s_{1}, s_{2}\right]$ where $s_{1}=k_{1}^{(1)} R_{\Lambda} / k_{\perp}^{2}$ and $s_{2}=k_{1}^{(2)} R_{\Lambda} / k_{\perp}^{2}$ where $k_{1}^{(1)}$ and $k_{1}^{(2)}$ are the two streamwise wave numbers computed using Taylor's hypothesis from the frequencies $f_{1}$ and $f_{2}$ that define the frequency band. 
Comparison with experimental results for three frequency bands is shown in Figs. 15(b)-15(d). Again the matching with experimental data is good for the open loop. It is clear that the largest contribution for the broadband rms originates from the smallest frequency band 1-4 Hz. For larger frequencies the contribution is smaller, and for all cases the controller performs well, but its effectiveness diminishes for larger frequencies.

Using Eq. (30), which is valid for every velocity component, and taking into account Eqs. (4) and (6), the instantaneous blowing or suction velocity $v_{w}$ can be obtained:

$$
v_{w}(x, z, t)=\int_{-\infty}^{+\infty} \int_{-\infty}^{+\infty} \int_{-\infty}^{+\infty} \frac{i k_{3}}{k_{1}}\left(\hat{u}_{3}^{\infty}+\frac{i k_{3}}{\gamma} \hat{u}_{2}^{\infty}\right)\left(\frac{2 \bar{x} k_{1}}{R_{\Lambda}}\right)^{1 / 2} \bar{v}\left(k_{1} x, 0\right) e^{i\left(k_{3} z-k_{1} t\right)} d k_{1} d k_{2} d k_{3} .
$$

Using the fact that $\bar{x}=k_{1} x$ and writing $\bar{v}\left(k_{1} x, 0\right)=\bar{v}_{w}(\bar{x})$ yields

$$
v_{w}(x, z, t)=\int_{-\infty}^{+\infty} \int_{-\infty}^{+\infty} \int_{-\infty}^{+\infty} \frac{i k_{3}}{k_{1}}\left(\hat{u}_{3}^{\infty}+\frac{i k_{3}}{\gamma} \hat{u}_{2}^{\infty}\right)\left(\frac{2 \bar{x} k_{1}}{R_{\Lambda}}\right)^{1 / 2} \bar{v}_{w}(\bar{x}) e^{i\left(k_{3} z-k_{1} t\right)} d k_{1} d k_{2} d k_{3} .
$$

Keeping only the feedback part of the actuation signal, i.e., $\bar{v}_{w}^{\prime}(\bar{x})=-\mathbf{K}_{i} \mathbf{q}_{i}$, one obtains $\bar{v}_{w}(\bar{x})=$ $-\int_{0}^{\bar{x}} \mathbf{K}(\tilde{x}) \mathbf{q}(\tilde{x}) d \tilde{x}$, where the initial condition $\bar{v}_{w}(0)=0$ was used. Substituting in the previous equation one finds

$$
\begin{aligned}
v_{w}(x, z, t)= & \int_{-\infty}^{+\infty} \int_{-\infty}^{+\infty} \int_{-\infty}^{+\infty} \frac{i k_{3}}{k_{1}}\left(\hat{u}_{3}^{\infty}+\frac{i k_{3}}{\gamma} \hat{u}_{2}^{\infty}\right)\left(\frac{2 \bar{x} k_{1}}{R_{\Lambda}}\right)^{1 / 2} \\
& \times\left[-\int_{0}^{\bar{x}} \mathbf{K}(\tilde{x}) \mathbf{q}(\tilde{x}) d \tilde{x}\right] e^{i\left(k_{3} z-k_{1} t\right)} d k_{1} d k_{2} d k_{3} .
\end{aligned}
$$

In the integral within the square brackets, $\left(k_{1}, k_{2}, k_{3}\right)$ are constant, so

$$
\begin{aligned}
v_{w}(x, z, t)= & \int_{-\infty}^{+\infty} \int_{-\infty}^{+\infty} \int_{-\infty}^{+\infty} \frac{i k_{3}}{k_{1}}\left(\frac{2 \bar{x} k_{1}}{R_{\Lambda}}\right)^{1 / 2} \\
& \times\left[-\int_{0}^{\bar{x}} \mathbf{K}(\tilde{x})\left(\hat{u}_{3}^{\infty}+\frac{i k_{3}}{\gamma} \hat{u}_{2}^{\infty}\right) \mathbf{q}(\tilde{x}) e^{i\left(k_{3} z-k_{1} t\right)} d \tilde{x}\right] d k_{1} d k_{2} d k_{3} .
\end{aligned}
$$

The product $\left(\hat{u}_{3}^{\infty}+i k_{3} \hat{u}_{2}^{\infty} / \gamma\right) \mathbf{q}(\tilde{x}) e^{i\left(k_{3} z-k_{1} t\right)}$ is just the instantaneous field [see Eqs. (4) and (6)], which is then multiplied by the control matrix. Strictly speaking, because the state q contains the coefficients of the Chebychev polynomials, one needs to multiply this vector with a constant coefficient matrix to obtain the velocity and pressure field at the collocation points. Of course, since the physical spatial location $x$ is kept constant and $k_{1}$ varies, results from different $\bar{x}$ need to be considered. In the above formulation we have minimized the cost function independently for each scaled wave number $\kappa, \kappa_{2}$. However, these wave numbers contain in their definition the streamwise wave number $k_{1}$, which also plays the role of temporal frequency due to Taylor's hypothesis. This may affect the causality of the derived controllers. In this work we have not investigated this, which may be the subject of future work.

All the analysis presented above is based on linearized equations. A next step would be the incorporation of the controller in a full Navier-Stokes solver to examine robustness to nonlinearity. Another step would be the development of a feedback controller directly for the nonlinear unsteady boundary-region equations, which describe the dynamics of nonlinear laminar streaks engendered by free-stream turbulence. This will pave the way to stabilize secondary instability and therefore control bypass transition.

\section{CONCLUSIONS}

The paper presents a rigorous approach to include the effect of free-stream turbulence in the formulation and the optimal control of laminar boundary layer streaks. It was shown that the 
randomness of free-stream turbulence at a particular wave number enters the system of equations as a multiplying factor of deterministic variables. The expectation of the objective function was obtained after integration in the wave number space. This operation naturally introduced the spectrum of free-stream turbulence in the control objective. The control gains obtained were independent of the spectrum, and in that sense universal. Furthermore, the interaction between the free-stream flow and the boundary layer flow introduced a forcing term on the system equations, which is independent of the states. The control problem was reformulated and solved, and we proved that the solution can be obtained analytically in terms of two sequences that can be computed by marching once backwards. It was found that the feedback part is by far the most important one; the feed-forward part makes only a small contribution to the minimal energy. This is an important conclusion because the feedback part is the one that depends on the flow variables, and can be computed from wall measurements.

It was also shown that, for appropriate weighting matrices, the adjoint equations and the controlled solution have self-similar behavior for large $\kappa$. The adjoint variables and the variables of the LUBR equations admit different self-similarity scalings. These were derived analytically and confirmed numerically. The developed method was applied to suppress the growth of streaks in a flat plate boundary layer. It was shown that the controller generates actuation velocity that opposes the wall-normal velocity in the near-wall region. It was also applied to control the full wave number spectrum of a real case where experimental data are available, and again it led to a reduction of the rms velocity for all frequency bands examined.

\section{ACKNOWLEDGMENT}

The authors would like to thank EPSRC (Grant No. EP/I016015/1) for funding this work. We would like also to thank the anonymous reviewer and Prof. Luchini for raising the causality issue when minimising independently over each temporal frequency.

\section{APPENDIX A: WHY DOES RANDOMNESS APPEAR MULTIPLICATIVELY?}

In the paper, randomness appears multiplicatively. This is quite unusual and warrants some explanation. Usually randomness appears additively as a stochastic body force on the right-hand side of an otherwise deterministic system and the independent variable is time (see, e.g., Refs. [44,45]). For the case examined in the paper we consider one particular frequency and the independent variable is $x$, so we would expect the form

$$
\mathcal{L} \frac{\partial \mathbf{q}}{\partial x}=\mathcal{M}(\mathbf{q})+w(x)
$$

where $w(x)$ is a random variable that depends on $x$. The variable $w(x)$ is considered to be white noise, that is, the expectation of the correlation at two different locations is proportional to the Dirac function, $\left\langle w(x) w\left(x^{\prime}\right)\right\rangle=W \delta\left(x-x^{\prime}\right)$. Note that the independent variable of the system $(x$ in the system above) is the same as the variable used to define the correlation $\left\langle w(x) w\left(x^{\prime}\right)\right\rangle$. The fact that the correlation is proportional to the delta function $\delta\left(x-x^{\prime}\right)$ means that $w(x)$ is uncorrelated at two different $x$ locations.

We examine more carefully how randomness enters our system. We will focus on regions I and II of Fig. 1 because they are crucial on determining how randomness is handled. We assume the perturbation solution 4 and substitute it in the Navier-Stokes equations. Assuming that $\epsilon$ is small and ignoring the nonlinear terms that scale as $\epsilon^{2}$ we obtain the linearized system:

$$
\mathcal{L}_{\mathrm{LUBL}} \frac{\partial \overline{\mathbf{q}}_{0}}{\partial \bar{x}}=\mathcal{M}\left(\overline{\mathbf{q}}_{0}\right)
$$

where $\overline{\mathbf{q}}_{0}=\left\{\begin{array}{llll}\bar{u}_{0} & \bar{v}_{0} & \bar{w}_{0} & \bar{p}_{0}\end{array}\right\}^{T}$ and $\mathcal{L}_{\text {LUBL }}$ is the linearized unsteady boundary-layer (LUBL) operator, i.e., Eqs. (4.5) and (4.6) in Ref. [23]). This operator applies the standard boundary layer approximation, i.e., it contains only wall normal derivatives and does not include pressure. At 
the wall, the boundary conditions are the no slip conditions. At the top of the boundary layer, the boundary condition is the velocity distribution given by the second term on the right-hand side of Eq. (3). This leads to the following boundary conditions as $\eta \rightarrow \infty$ :

$$
\begin{aligned}
\epsilon \bar{u}_{0} e^{i\left(k_{3} z-k_{1} t\right)} & =\epsilon u_{1}^{(1)} e^{i\left[k_{1}(x-t)+k_{3} z\right]} \Rightarrow \bar{u}_{0}=u_{1}^{(1)} e^{i \bar{x}}=\left[\hat{u}_{1}^{\infty}+\left(i k_{1} / \gamma\right) \hat{u}_{2}^{\infty}\right] e^{i \bar{x}}, \\
\epsilon \bar{w}_{0} e^{i\left(k_{3} z-k_{1} t\right)} & =\epsilon u_{3}^{(1)} e^{i\left[k_{1}(x-t)+k_{3} z\right]} \Rightarrow \bar{w}_{0}=u_{3}^{(1)} e^{i \bar{x}}=\left[\hat{u}_{3}^{\infty}+\left(i k_{3} / \gamma\right) \hat{u}_{2}^{\infty}\right] e^{i \bar{x}} .
\end{aligned}
$$

These boundary conditions force the linear system at the top. The randomness appears in the Fourier coefficients $\hat{u}_{1}^{\infty}, \hat{u}_{2}^{\infty}, \hat{u}_{3}^{\infty}$, that depend only on the wave number vector, $\mathbf{k}$, and not on $\bar{x}$. It is important to recognize that the randomness is now uncorrelated not in the physical space (i.e., the $\bar{x}$ direction) but in the wave number space because $\left\langle\hat{u}_{i}^{\infty}(\mathbf{k}) \hat{u}_{j}^{\infty \star}\left(\mathbf{k}^{\prime}\right)\right\rangle=\Phi_{\infty i j}(\mathbf{k}) \delta\left(\mathbf{k}-\mathbf{k}^{\prime}\right)$ where $\Phi_{\infty i j}$ is the spectral tensor of free-stream turbulence. It is possible to incorporate these boundary conditions in system (A2). This is more easily understood if we discretize the system in the $\eta$ direction. Using the superscript $\delta$ to denote the discrete form of the operators we get

$$
\mathcal{L}_{\mathrm{LUBL}}^{\delta} \frac{\delta \overline{\mathbf{q}}_{0}}{\delta \bar{x}}=\mathcal{M}^{\delta}\left(\overline{\mathbf{q}}_{0}\right)+w\left(\hat{u}_{1}^{\infty}, \hat{u}_{2}^{\infty}, \hat{u}_{3}^{\infty}\right) e^{i \bar{x}} .
$$

The vector $w\left(\hat{u}_{1}^{\infty}, \hat{u}_{2}^{\infty}, \hat{u}_{3}^{\infty}\right)$ is zero everywhere apart from two rows that express the boundary conditions at the top of the boundary layer for the $\bar{x}$ and $z$ momentum equations (at these rows this vector is equal to $u_{1}^{(1)}$ and $u_{3}^{(1)}$ respectively). In this equation, the randomness does appear additively (as usually). There is, however, a fundamental difference between (A1) and (A4): the random coefficients $u_{1}^{(1)}, u_{3}^{(1)}$ [from which $w\left(\hat{u}_{1}^{\infty}, \hat{u}_{2}^{\infty}, \hat{u}_{3}^{\infty}\right)$ is computed] are independent of $\bar{x}$ and due to the linearity they can be factored out. Therefore the solution of (A4) can be written as a linear combination of solutions as (see also Ref. [23])

$$
\begin{aligned}
\left\{\bar{u}_{0}, \bar{v}_{0}, \bar{w}_{0}\right\} & =u_{3}^{(1)}\left\{\frac{i k_{3}}{k_{1}} \bar{u}, \frac{i k_{3}}{k_{1}} \bar{v}, \bar{w}\right\}+u_{1}^{(1)}\left\{\bar{u}^{(0)}, \bar{v}^{(0)}, 0\right\} \\
& =\left(\hat{u}_{3}^{\infty}+\frac{i k_{3}}{\gamma} \hat{u}_{2}^{\infty}\right)\left\{\frac{i k_{3}}{k_{1}} \bar{u}, \frac{i k_{3}}{k_{1}} \bar{v}, \bar{w}\right\}+\left(\hat{u}_{1}^{\infty}+\frac{i k_{3}}{\gamma} \hat{u}_{2}^{\infty}\right)\left\{\bar{u}^{(0)}, \bar{v}^{(0)}, 0\right\} .
\end{aligned}
$$

The coefficient $i k_{3} / k_{1}$ has been added to simplify the resulting equations for $\bar{u}, \bar{v}, \bar{w}$. In the LUBL system,the $z$-momentum equation is decoupled from the others and is forced only from $u_{3}^{(1)}$, and for this reason the coefficient corresponding to forcing from $u_{1}^{(1)}$ is zero. Only the first term is important in the core of the boundary layer and is retained in the present work. Therefore we finally have

$$
\left\{\bar{u}_{0}, \bar{v}_{0}, \bar{w}_{0}\right\}=\left(\hat{u}_{3}^{\infty}+\frac{i k_{3}}{\gamma} \hat{u}_{2}^{\infty}\right)\left\{\frac{i k_{3}}{k_{1}} \bar{u}, \frac{i k_{3}}{k_{1}} \bar{v}, \bar{w}\right\} .
$$

This representation is also retained in the LUBR equations, which are valid in region III. In this region, the pressure also appears and the form of the solution is Eq. (6):

$$
\left\{\bar{u}_{0}, \bar{v}_{0}, \bar{w}_{0}, \bar{p}_{0}\right\}=\left(\hat{u}_{3}^{\infty}+\frac{i k_{3}}{\gamma} \hat{u}_{2}^{\infty}\right)\left\{\frac{i k_{3}}{k_{1}} \bar{u}, \frac{i k_{3}}{k_{1}} \bar{v}, \bar{w}, i \kappa\left(\frac{k_{1}}{R_{\Lambda}}\right)^{1 / 2} \bar{p}\right\} .
$$

The previous analysis explains why the randomness appears multiplicatively. In the formulation of the cost function to be minimized by the control effort, this naturally leads to the appearance of the spectrum function [Eq. (37)].

\section{APPENDIX B: DISCRETIZATION OF THE LUBR EQUATIONS AND CODE VALIDATION}

In this Appendix, the discretization of the LUBR equations and the code validation are presented. In order to minimize the number of states, their distributions in the wall-normal direction $\eta$ are projected into a series of rational Chebyshev polynomials [46]. The standard Chebyshev polynomials 
$\Gamma_{n}(y)$ are defined in the interval $-1 \leqslant y \leqslant 1$. In order to use these polynomials in the interval $0 \leqslant$ $\eta \leqslant \eta_{\max }$ required in the boundary layer, an algebraic mapping is used that clusters the collocation points in the near-wall region:

$$
\eta=\frac{c(1+y)}{d-y}
$$

where

$$
c=\frac{\eta_{\operatorname{mid}} \eta_{\max }}{\eta_{\max }-2 \eta_{\operatorname{mid}}} \quad \text { and } \quad d=1+\frac{2 c}{\eta_{\max }} .
$$

This mapping places half of the collocation points in the region $0 \leqslant \eta \leqslant \eta_{\text {mid }}$. The variables $\bar{u}, \bar{v}, \bar{w}$, and $\bar{p}$ are then projected to a finite number of $N+1$ rational Chebyshev polynomials as

$$
\left[\begin{array}{c}
\bar{u} \\
\bar{v} \\
\bar{w} \\
\bar{p}
\end{array}\right](\bar{x}, \eta)=\sum_{n=0}^{N}\left[\begin{array}{c}
a_{\bar{u}, n}(\bar{x}) \\
a_{\bar{v}, n}(\bar{x}) \\
a_{\bar{w}, n}(\bar{x}) \\
a_{\bar{p}, n}(\bar{x})
\end{array}\right] \Gamma_{n}(\eta) .
$$

The equations are discretized on a grid of collocation points, $\eta\left(y_{k}\right)$, where $y_{k}=\cos (\pi k / N), k=$ $0, \ldots, N$. In our code, the continuity equation is solved directly (rather than converting it to a Poisson equation for pressure). References $[47,48]$ provide a detailed analysis on the application of spectral methods to inhomogeneous flows and the treatment of pressure.

After the spatial discretization of the LUBR equations in the wall-normal direction, the system can be written as

$$
\mathbf{L} \frac{d \hat{\mathbf{q}}}{d \bar{x}}=\mathbf{M} \hat{\mathbf{q}}+\mathbf{G}
$$

where vector $\hat{\mathbf{q}}$ contains the coefficients of velocity and pressure,

$$
\hat{\mathbf{q}}=\left[a_{\bar{u}, 0} \cdots a_{\bar{u}, N}, a_{\bar{v}, 0} \cdots a_{\bar{v}, N}, a_{\bar{w}, 0} \cdots a_{\bar{w}, N}, a_{\bar{p}, 0} \cdots a_{\bar{p}, N}\right]^{T}
$$

and is the state vector. The matrices $\mathbf{L}$ and $\mathbf{M}$ contain the coefficients of the unknown variables corresponding to the LUBR equations, and the vector $\mathbf{G}$ contains the effect of external forcing from free-stream turbulence.

The developed numerical model is validated against the results from Ref. [23]. The mapping variables are set as $\eta_{\text {mid }}=4$ and $\eta_{\max }=15$. These values provide good resolution of the boundary layer close to the wall without placing many collocation points in the free stream where the variation is smooth. Different values of the number of collocation points $N$ were tested; for $N>50$ grid independent results are obtained. Different values of $\Delta \bar{x}$ were also tested, and we found that $\Delta \bar{x}=0.001$ gave grid independent results, in close agreement with the reference data of Ref. [23] as shown in Fig. 16. The asymptotic behavior of the solution for $\kappa \gg 1$ has also been verified (not shown for brevity).

\section{APPENDIX C: DERIVATION OF WALL-BASED OPTIMAL CONTROL}

In this Appendix, the wall-based optimal controller is derived. In the following derivations, the "matrix inversion lemma" will be applied several times. The lemma states that if $\mathbf{A}, \mathbf{C}$ and $\mathbf{C}^{-1}+\mathbf{D A}^{-1} \mathbf{B}$ are general nonsingular square matrices $[\mathbf{A}, \mathbf{B}$, and $\mathbf{C}$ are not to be confused with $\mathbf{A}_{i}, \mathbf{B}_{i}$, and $\mathbf{C}_{i}$ in Eq. (26)], then $\mathbf{A}+\mathbf{B C D}$ is invertible and

$$
(\mathbf{A}+\mathbf{B C D})^{-1}=\mathbf{A}^{-1}-\mathbf{A}^{-1} \mathbf{B}\left(\mathbf{C}^{-1}+\mathbf{D A} \mathbf{A}^{-1} \mathbf{B}\right)^{-1} \mathbf{D} \mathbf{A}^{-1} .
$$

The lemma can be proved easily by multiplying A + BCD by the right-hand side of (C1). Substitution of (50) into the stationarity condition (46), the control signal becomes

$$
\mathbf{u}_{i}=-\mathbf{R}_{i}^{-1} \mathbf{B}_{i}^{\star}\left(\mathbf{P}_{i+1} \mathbf{q}_{i+1}-\mathbf{V}_{i+1}\right) .
$$



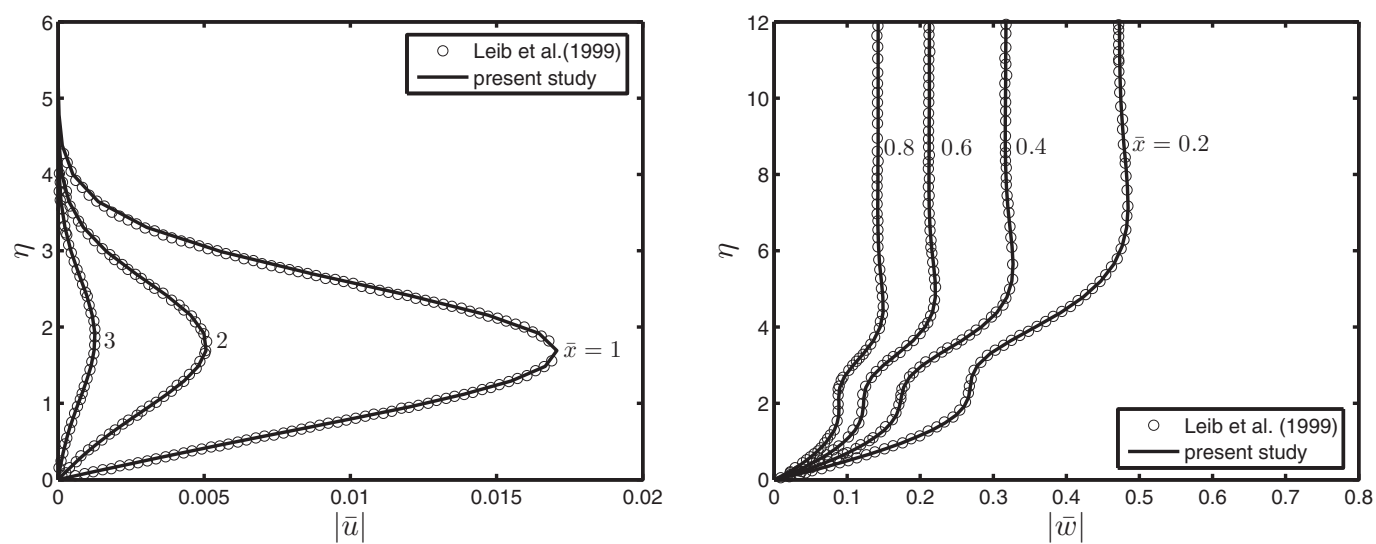

FIG. 16. Profiles of the magnitudes of the (left) streamwise and (right) spanwise perturbation velocity at various values of $\bar{x}$ for $\kappa=1$ and $\kappa_{2}=-1$.

Substitution of (C2) into the state equation (44) and solving for $\mathbf{q}_{i+1}$ one finds

$$
\mathbf{q}_{i+1}=\left(\mathbf{I}+\mathbf{B}_{i} \mathbf{R}_{i}^{-1} \mathbf{B}_{i}^{\star} \mathbf{P}_{i+1}\right)^{-1}\left(\mathbf{A}_{i} \mathbf{q}_{i}+\mathbf{B}_{i} \mathbf{R}_{i}^{-1} \mathbf{B}_{i}^{\star} \mathbf{V}_{i+1}+\mathbf{C}_{i}\right) .
$$

Substitution of (50) and (C3) into the adjoint equation (45) leads to

$$
\begin{aligned}
0= & {\left[-\mathbf{P}_{i}+\mathbf{A}_{i}^{\star} \mathbf{P}_{i+1}\left(\mathbf{I}+\mathbf{B}_{i} \mathbf{R}_{i}^{-1} \mathbf{B}_{i}^{\star} \mathbf{P}_{i+1}\right)^{-1} \mathbf{A}_{i}+\mathbf{Q}_{i}\right] \mathbf{q}_{i} } \\
& +\left[\mathbf{V}_{i}+\mathbf{A}_{i}^{\star} \mathbf{P}_{i+1}\left(\mathbf{I}+\mathbf{B}_{i} \mathbf{R}_{i}^{-1} \mathbf{B}_{i}^{\star} \mathbf{P}_{i+1}\right)^{-1}\left(\mathbf{B}_{i} \mathbf{R}_{i}^{-1} \mathbf{B}_{i}^{\star} \mathbf{V}_{i+1}+\mathbf{C}_{i}\right)-\mathbf{A}_{i}^{\star} \mathbf{V}_{i+1}\right] .
\end{aligned}
$$

This equation must hold for all state sequences $\mathbf{q}_{i}$ given any $\mathbf{q}_{0}$. Therefore the terms inside the square brackets must individually vanish. Using the matrix inversion lemma,

$$
\left(\mathbf{I}+\mathbf{B}_{i} \mathbf{R}_{i}^{-1} \mathbf{B}_{i}^{\star} \mathbf{P}_{i+1}\right)^{-1}=\mathbf{I}-\mathbf{B}_{i}\left(\mathbf{R}_{i}+\mathbf{B}_{i}^{\star} \mathbf{P}_{i+1} \mathbf{B}_{i}\right)^{-1} \mathbf{B}_{i}^{\star} \mathbf{P}_{i+1},
$$

the discrete algebraic Riccati equation for $\mathbf{P}_{i}$ can be written as

$$
\mathbf{P}_{i}=\mathbf{A}_{i}^{\star} \mathbf{P}_{i+1} \mathbf{A}_{i}+\mathbf{Q}_{i}-\mathbf{A}_{i}^{\star} \mathbf{P}_{i+1} \mathbf{B}_{i}\left(\mathbf{R}_{i}+\mathbf{B}_{i}^{\star} \mathbf{P}_{i+1} \mathbf{B}_{i}\right)^{-1} \mathbf{B}_{i}^{\star} \mathbf{P}_{i+1} \mathbf{A}_{i} .
$$

If the feedback gain is defined as

$$
\mathbf{K}_{i}=\left(\mathbf{R}_{i}+\mathbf{B}_{i}^{\star} \mathbf{P}_{i+1} \mathbf{B}_{i}\right)^{-1} \mathbf{B}_{i}^{\star} \mathbf{P}_{i+1} \mathbf{A}_{i}
$$

the equation for $\mathbf{P}_{i}$ becomes

$$
\mathbf{P}_{i}=\mathbf{A}_{i}^{\star} \mathbf{P}_{i+1}\left(\mathbf{A}_{i}-\mathbf{B}_{i} \mathbf{K}_{i}\right)+\mathbf{Q}_{i}
$$

The equation for $\mathbf{V}_{i}$ becomes

$$
\mathbf{V}_{i}=\mathbf{A}_{i}^{\star}\left\{\mathbf{V}_{i+1}-\mathbf{P}_{i+1}\left[\left(\mathbf{I}+\mathbf{B}_{i} \mathbf{R}_{i}^{-1} \mathbf{B}_{i}^{\star} \mathbf{P}_{i+1}\right)^{-1} \mathbf{B}_{i} \mathbf{R}_{i}^{-1} \mathbf{B}_{i}^{\star} \mathbf{V}_{i+1}+\left(\mathbf{I}+\mathbf{B}_{i} \mathbf{R}_{i}^{-1} \mathbf{B}_{i}^{\star} \mathbf{P}_{i+1}\right)^{-1} \mathbf{C}_{i}\right]\right\} .
$$

Using the matrix inversion lemma the term $\left(\mathbf{I}+\mathbf{B}_{i} \mathbf{R}_{i}^{-1} \mathbf{B}_{i}^{\star} \mathbf{P}_{i+1}\right)^{-1} \mathbf{B}_{i} \mathbf{R}_{i}^{-1} \mathbf{B}_{i}^{\star} \mathbf{V}_{i+1}$ can be written as

$$
\begin{aligned}
\left(\mathbf{I}+\mathbf{B}_{i} \mathbf{R}_{i}^{-1} \mathbf{B}_{i}^{\star} \mathbf{P}_{i+1}\right)^{-1} \mathbf{B}_{i} \mathbf{R}_{i}^{-1} \mathbf{B}_{i}^{\star} \mathbf{V}_{i+1} & =\left[\mathbf{I}-\mathbf{B}_{i}\left(\mathbf{R}_{i}+\mathbf{B}_{i}^{\star} \mathbf{P}_{i+1} \mathbf{B}_{i}\right)^{-1} \mathbf{B}_{i}^{\star} \mathbf{P}_{i+1}\right] \mathbf{B}_{i} \mathbf{R}_{i}^{-1} \mathbf{B}_{i}^{\star} \mathbf{V}_{i+1} \\
& =\mathbf{B}_{i}\left[\mathbf{R}_{i}^{-1}-\left(\mathbf{R}_{i}+\mathbf{B}_{i}^{\star} \mathbf{P}_{i+1} \mathbf{B}_{i}\right)^{-1} \mathbf{B}_{i}^{\star} \mathbf{P}_{i+1} \mathbf{B}_{i} \mathbf{R}_{i}^{-1}\right] \mathbf{B}_{i}^{\star} \mathbf{V}_{i+1} .
\end{aligned}
$$

Using the matrix inversion lemma once more, the term within square brackets can be simplified as

$$
\mathbf{R}_{i}^{-1}-\left(\mathbf{R}_{i}+\mathbf{B}_{i}^{\star} \mathbf{P}_{i+1} \mathbf{B}_{i}\right)^{-1} \mathbf{B}_{i}^{\star} \mathbf{P}_{i+1} \mathbf{B}_{i} \mathbf{R}_{i}^{-1}=\left(\mathbf{R}_{i}+\mathbf{B}_{i}^{\star} \mathbf{P}_{i+1} \mathbf{B}_{i}\right)^{-1},
$$


and (C9) becomes

$$
\mathbf{V}_{i}=\mathbf{A}_{i}^{\star}\left\{\mathbf{V}_{i+1}-\mathbf{P}_{i+1} \mathbf{B}_{i}\left(\mathbf{R}_{i}+\mathbf{B}_{i}^{\star} \mathbf{P}_{i+1} \mathbf{B}_{i}\right)^{-1} \mathbf{B}_{i}^{\star} \mathbf{V}_{i+1}+\mathbf{P}_{i+1}\left[\mathbf{I}-\mathbf{B}_{i}\left(\mathbf{R}_{i}+\mathbf{B}_{i}^{\star} \mathbf{P}_{i+1} \mathbf{B}_{i}\right)^{-1} \mathbf{B}_{i}^{\star} \mathbf{P}_{i+1}\right] \mathbf{C}_{i}\right\} .
$$

Noting that

$$
\left(\mathbf{A}_{i}-\mathbf{B}_{i} \mathbf{K}_{i}\right)^{\star}=\mathbf{A}_{i}^{\star}-\mathbf{K}_{i}^{\star} \mathbf{B}_{i}^{\star}=\mathbf{A}_{i}^{\star}-\mathbf{A}_{i}^{\star} \mathbf{P}_{i+1} \mathbf{B}_{i}\left(\mathbf{R}_{i}+\mathbf{B}_{i}^{\star} \mathbf{P}_{i+1} \mathbf{B}_{i}\right)^{-1} \mathbf{B}_{i}^{\star},
$$

where the fact that $\mathbf{P}_{i+1}$ is Hermitian was employed ( $\left.\mathbf{P}_{i+1}^{\star}=\mathbf{P}_{i+1}\right)$, Eq. (C11) becomes

$$
\mathbf{V}_{i}=\left(\mathbf{A}_{i}-\mathbf{B}_{i} \mathbf{K}_{i}\right)^{\star} \mathbf{V}_{i+1}-\left(\mathbf{A}_{i}-\mathbf{B}_{i} \mathbf{K}_{i}\right)^{\star} \mathbf{P}_{i+1} \mathbf{C}_{i}
$$

or

$$
\mathbf{V}_{i}=\left(\mathbf{A}_{i}-\mathbf{B}_{i} \mathbf{K}_{i}\right)^{\star}\left(\mathbf{V}_{i+1}-\mathbf{P}_{i+1} \mathbf{C}_{i}\right) .
$$

Since the sequences $\mathbf{P}_{i}$ and $\mathbf{V}_{i}$ can be calculated, assumption (50) is valid. Comparing (48) and (50), the boundary condition for $\mathbf{V}_{i}$ at the end of the domain is

$$
\mathbf{V}_{N}=0 \text {. }
$$

The control signal is computed as follows. Substitution of (44) into (C2) leads to

$$
\mathbf{u}_{i}=-\mathbf{R}_{i}^{-1} \mathbf{B}_{i}^{\star} \mathbf{P}_{i+1}\left(\mathbf{A}_{i} \mathbf{q}_{i}+\mathbf{B}_{i} \mathbf{u}_{i}+\mathbf{C}_{i}\right)+\mathbf{R}_{i}^{-1} \mathbf{B}_{i}^{\star} \mathbf{V}_{i+1} .
$$

This equation is implicit in $\mathbf{u}_{i}$. Multiplying from the left with $\mathbf{R}_{i}$ and solving for $\mathbf{u}_{i}$ one finds

$$
\mathbf{u}_{i}=-\left(\mathbf{R}_{i}+\mathbf{B}_{i}^{\star} \mathbf{P}_{i+1} \mathbf{B}_{i}\right)^{-1} \mathbf{B}_{i}^{\star} \mathbf{P}_{i+1} \mathbf{A}_{i} \mathbf{q}_{i}+\left(\mathbf{B}_{i}^{\star} \mathbf{P}_{i+1} \mathbf{B}_{i}+\mathbf{R}_{i}\right)^{-1} \mathbf{B}_{i}^{\star}\left(\mathbf{V}_{i+1}-\mathbf{P}_{i+1} \mathbf{C}_{i}\right)
$$

If we define the feed-forward gain as

$$
\mathbf{K}_{i}^{v}=\left(\mathbf{B}_{i}^{\star} \mathbf{P}_{i+1} \mathbf{B}_{i}+\mathbf{R}_{i}\right)^{-1} \mathbf{B}_{i}^{\star},
$$

and using (C7) for the feedback gain, the optimal control signal takes the final form:

$$
\mathbf{u}_{i}=-\mathbf{K}_{i} \mathbf{q}_{i}+\mathbf{K}_{i}^{v}\left(\mathbf{V}_{i+1}-\mathbf{P}_{i+1} \mathbf{C}_{i}\right)
$$

\section{APPENDIX D: FUNDAMENTAL PERFORMANCE LIMIT}

In this Appendix, an explicit expression is derived for the a priori computation of the minimum value of the objective function, $J_{\min }$ by use of only the system matrices as well as the free-stream and initial conditions. The state and adjoint variables do not enter this expression. The derivation is based on the proof presented in Ref. [37]. Assume the dynamical system

$$
\mathbf{x}_{i+1}=\mathbf{A}_{i} \mathbf{x}_{i}+\mathbf{B}_{i} \mathbf{u}_{i} \quad(i=0 \ldots N-1)
$$

and a cost function that takes the form of sum of inner products in complex space:

$$
J_{1}=\sum_{i=0}^{N} \mathbf{y}_{i}^{\star} \mathbf{x}_{i}=\sum_{i=0}^{N} \overline{\mathbf{x}_{i}^{\star} \mathbf{y}_{i}},
$$

where $\mathbf{y}_{i}^{\star}$ are weighting vectors. The adjoint of the system with respect to this cost function is

$$
\lambda_{i}=\mathbf{A}_{i}^{\star} \lambda_{i+1}+\mathbf{y}_{i}, \quad \lambda_{N}=\mathbf{y}_{N},
$$

and $J_{1}$ can be written in terms of the adjoint variables as

$$
J_{1}=\lambda_{0}^{\star} \mathbf{x}_{0}+\sum_{i=0}^{N-1} \lambda_{i+1}^{\star}\left(\mathbf{B}_{i} \mathbf{u}_{i}\right)
$$


This expression shows that the adjoint variables $\lambda_{0}^{\star}$ and $\lambda_{i+1}^{\star}$ can be thought of as the sensitivities of the cost function to the inputs of the dynamical system, $\mathbf{x}_{0}$ and $\mathbf{B}_{i} \mathbf{u}_{i}$, respectively. If the cost function is defined as

$$
J_{2}=\overline{J_{1}}=\sum_{i=0}^{N} \mathbf{x}_{i}^{\star} \mathbf{y}_{i}
$$

then the adjoint equation remains the same, and it can be easily proven that $J_{2}$ can be written as

$$
J_{2}=\mathbf{x}_{0}^{\star} \lambda_{0}+\sum_{i=0}^{N-1}\left(\mathbf{B}_{i} \mathbf{u}_{i}\right)^{\star} \lambda_{i+1} .
$$

In the present case, the dynamical system is given by (44), but the cost function (41) is quadratic in terms of the state vector q. However, it is still possible to write the cost function in terms of the adjoint variables. The process is the following. We take $\mathbf{y}_{i}=\mathbf{q}_{i} \mathbf{q}_{i}$, use the fact that $\mathbf{q}_{i}$ is Hermitian and apply the same procedure used to derive (D4). After some algebra one finds

$$
J=\frac{1}{2} \lambda_{0}^{\star} \mathbf{q}_{0}+\frac{1}{2} \sum_{i=0}^{N-1} \lambda_{i+1}^{\star}\left(\mathbf{B}_{i} \mathbf{u}_{i}+\mathbf{C}_{i}\right)+\frac{1}{2} \sum_{i=0}^{N-1} \mathbf{u}_{i}^{\star} \mathbf{R}_{i} \mathbf{u}_{i}
$$

or, after some rearrangement,

$$
J=\frac{1}{2} \lambda_{0}^{\star} \mathbf{q}_{0}+\frac{1}{2} \sum_{i=0}^{N-1}\left(\lambda_{i+1}^{\star} \mathbf{B}_{i}+\mathbf{u}_{i}^{\star} \mathbf{R}_{i}\right) \mathbf{u}_{i}+\frac{1}{2} \sum_{i=0}^{N-1} \lambda_{i+1}^{\star} \mathbf{C}_{i} .
$$

Using the optimality condition (47) the second term on the right-hand side vanishes and the equation takes the simplified form:

$$
J_{\min }=\frac{1}{2} \lambda_{0}^{\star} \mathbf{q}_{0}+\frac{1}{2} \sum_{i=0}^{N-1} \lambda_{i+1}^{\star} \mathbf{C}_{i} .
$$

It is clear then that the variables $(1 / 2) \lambda_{0}^{\star}$ and $(1 / 2) \lambda_{i+1}^{\star}$ denote physically the sensitivity of $J_{\min }$ to the inputs of the open loop system, $\mathbf{q}_{0}$ and $\mathbf{C}_{i}$, respectively.

If the external forcing $\mathbf{C}_{i}=\mathbf{0}$, then $\lambda_{i}=\mathbf{P}_{i} \mathbf{q}_{i}$, and the well-known result $J_{\min }=\frac{1}{2} \lambda_{0}^{\star} \mathbf{q}_{0}=$ $\frac{1}{2} \mathbf{q}_{0}^{\star} \mathbf{P}_{0} \mathbf{q}_{0}$ is obtained [36]. In that case, $J_{\text {min }}$ can be computed using only the initial conditions $\mathbf{q}_{0}$ and $\mathbf{P}_{0}$ recursively from (C6), an expression that contains only the matrices of the system. When $\mathbf{C}_{i}$ is present, the analysis is more involved. To simplify the notation, we denote explicitly the feedback and feed-forward parts of the control variable in Eq. (51):

$$
\mathbf{u}_{i}=-\mathbf{K}_{i} \mathbf{q}_{i}+\mathbf{u}_{\mathbf{f f} i}
$$

where $\mathbf{u}_{\mathbf{f f} i}$ denotes the feed-forward part:

$$
\mathbf{u}_{\mathbf{f f} i}=\mathbf{K}_{i}^{v}\left(\mathbf{V}_{i+1}-\mathbf{P}_{i+1} \mathbf{C}_{i}\right)
$$

The dynamical system for the closed loop plant now is

$$
\mathbf{q}_{i+1}=\left(\mathbf{A}_{i}-\mathbf{B}_{i} \mathbf{K}_{i}\right) \mathbf{q}_{i}+\mathbf{B}_{i} \mathbf{u}_{\mathbf{f f} i}+\mathbf{C}_{i} .
$$

Note that we have incorporated the feedback part of the control variable $-\mathbf{K}_{i} \mathbf{q}_{i}$ with the system matrix (as it is customary), while the feed-forward part and $\mathbf{C}_{i}$ result in forcing to the closed loop system. This means that the closed loop system has now as inputs the initial conditions $\mathbf{q}_{0}$ as well as the forcing $\mathbf{B}_{i} \mathbf{u}_{\mathbf{f f}} i+\mathbf{C}_{i}$. We anticipate then the presence of another adjoint variable that will quantify the sensitivity to those inputs.

Applying Eq. (50) at $i+1$ one finds

$$
\lambda_{i+1}=\mathbf{P}_{i+1}\left[\left(\mathbf{A}_{i}-\mathbf{B}_{i} \mathbf{K}_{i}\right) \mathbf{q}_{i}+\mathbf{B}_{i} \mathbf{u}_{\mathbf{f f} i}+\mathbf{C}_{i}\right]-\mathbf{V}_{i+1},
$$


and, substituting in Eq. (D9),

$$
\begin{aligned}
J_{\min } & =\frac{1}{2} \lambda_{0}^{\star} \mathbf{q}_{0}+\frac{1}{2} \sum_{i=0}^{N-1}\left\{\left[\left(\mathbf{A}_{i}-\mathbf{B}_{i} \mathbf{K}_{i}\right) \mathbf{q}_{i}+\mathbf{B}_{i} \mathbf{u}_{\mathbf{f f} i}+\mathbf{C}_{i}\right]^{\star} \mathbf{P}_{i+1}-\mathbf{V}_{i+1}^{\star}\right\} \mathbf{C}_{i} \\
& =\frac{1}{2} \lambda_{0}^{\star} \mathbf{q}_{0}+\frac{1}{2} \sum_{i=0}^{N-1} \mathbf{q}_{i}^{\star}\left(\mathbf{A}_{i}-\mathbf{B}_{i} \mathbf{K}_{i}\right)^{\star} \mathbf{P}_{i+1} \mathbf{C}_{i}+\frac{1}{2} \sum_{i=0}^{N-1}\left[\left(\mathbf{B}_{i} \mathbf{u}_{\mathbf{f f} i}+\mathbf{C}_{i}\right)^{\star} \mathbf{P}_{i+1} \mathbf{C}_{i}-\mathbf{V}_{i+1}^{\star} \mathbf{C}_{i}\right] .
\end{aligned}
$$

The first and third term in the above expression can be determined using the system matrices and the initial and free-stream boundary conditions; only the second term contains the states $\mathbf{q}_{i}$. In order to compute the second term (that defines a linear cost function) we apply the result mentioned at the beginning of this appendix using now the closed loop plant (D12) as the dynamical system and the weighting $\mathbf{y}_{i}=\left(\mathbf{A}_{i}-\mathbf{B}_{i} \mathbf{K}_{i}\right)^{\star} \mathbf{P}_{i+1} \mathbf{C}_{i}$. This defines the adjoint equation

$$
\boldsymbol{\xi}_{i}=\left(\mathbf{A}_{i}-\mathbf{B}_{i} \mathbf{K}_{i}\right)^{\star} \boldsymbol{\xi}_{i+1}+\left(\mathbf{A}_{i}-\mathbf{B}_{i} \mathbf{K}_{i}\right)^{\star} \mathbf{P}_{i+1} \mathbf{C}_{i}=\left(\mathbf{A}_{i}-\mathbf{B}_{i} \mathbf{K}_{i}\right)^{\star}\left(\boldsymbol{\xi}_{i+1}+\mathbf{P}_{i+1} \mathbf{C}_{i}\right)
$$

$(i=0 \ldots N-2)$ with terminal condition $\boldsymbol{\xi}_{N-1}=\left(\mathbf{A}_{N-1}-\mathbf{B}_{N-1} \mathbf{K}_{N-1}\right)^{\star} \mathbf{P}_{N} \mathbf{C}_{N-1}$. Using (D6) to express the linear cost function in terms of the adjoint variables, $J_{\min }$ becomes

$$
J_{\min }=\frac{1}{2} \lambda_{0}^{\star} \mathbf{q}_{0}+\frac{1}{2} \mathbf{q}_{0}^{\star} \boldsymbol{\xi}_{0}+\frac{1}{2} \sum_{i=0}^{N-2}\left(\mathbf{B}_{i} \mathbf{u}_{\mathbf{f f} i}+\mathbf{C}_{i}\right)^{\star} \boldsymbol{\xi}_{i+1}+\frac{1}{2} \sum_{i=0}^{N-1}\left[\left(\mathbf{B}_{i} \mathbf{u}_{\mathbf{f f} i}+\mathbf{C}_{i}\right)^{\star} \mathbf{P}_{i+1} \mathbf{C}_{i}-\mathbf{V}_{i+1}^{\star} \mathbf{C}_{i}\right]
$$

It is clear then that the adjoint variables $(1 / 2) \boldsymbol{\xi}_{0}$ and $(1 / 2) \boldsymbol{\xi}_{i+1}$ denote physically the sensitivity of the linear cost function $\sum_{i=0}^{N-1} \mathbf{q}_{i}^{\star}\left(\mathbf{A}_{i}-\mathbf{B}_{i} \mathbf{K}_{i}\right)^{\star} \mathbf{P}_{i+1} \mathbf{C}_{i}$ to the inputs of the closed loop system, $\mathbf{q}_{0}$ and $\mathbf{B}_{i} \mathbf{u}_{\mathbf{f f} i}+\mathbf{C}_{i}$, respectively.

Comparing Eqs. (D15) and (C14) it is noted that $\xi_{i}=-\mathbf{V}_{i}(i=0 \ldots N-1)$. This is also consistent with the boundary conditions of sequences $\boldsymbol{\xi}_{i}$ and $\mathbf{V}_{i}$. Indeed, using (C15), and substituting in Eq. (C14), one finds

$$
\mathbf{V}_{N-1}=-\left(\mathbf{A}_{N-1}-\mathbf{B}_{N-1} \mathbf{K}_{N-1}\right)^{\star} \mathbf{P}_{N} \mathbf{C}_{N-1}=-\boldsymbol{\xi}_{N-1} .
$$

The sequence $\mathbf{V}_{i}$ now has a direct physical meaning: it is the negative of the adjoint of the closed loop plant with respect to the cost function $\sum_{i=0}^{N-1} \mathbf{q}_{i}^{\star}\left(\mathbf{A}_{i}-\mathbf{B}_{i} \mathbf{K}_{i}\right)^{\star} \mathbf{P}_{i+1} \mathbf{C}_{i}$. Replacing $\boldsymbol{\xi}_{i}$ with $-\mathbf{V}_{i}$ leads to

$$
J_{\min }=\frac{1}{2} \mathbf{q}_{0}^{\star} \mathbf{P}_{0} \mathbf{q}_{0}-\frac{1}{2}\left(\mathbf{V}_{0}^{\star} \mathbf{q}_{0}+\mathbf{q}_{0}^{\star} \mathbf{V}_{0}\right)+\frac{1}{2} \sum_{i=0}^{N-1} \mathbf{W}_{i},
$$

where the sequence $\mathbf{W}_{i}$ is computed from the following expression by marching backwards:

$$
\begin{aligned}
\mathbf{W}_{i}= & -\left(\mathbf{V}_{i+1}-\mathbf{P}_{i+1} \mathbf{C}_{i}\right)^{\star} \mathbf{B}_{i}\left[\left(\mathbf{B}_{i}^{\star} \mathbf{P}_{i+1} \mathbf{B}_{i}+\mathbf{R}_{i}\right)^{\star}\right]^{-1} \mathbf{B}_{i}^{\star}\left(\mathbf{V}_{i+1}-\mathbf{P}_{i+1} \mathbf{C}_{i}\right) \\
& -\left(\mathbf{C}_{i}^{\star} \mathbf{V}_{i+1}+\mathbf{V}_{i+1}^{\star} \mathbf{C}_{i}\right)+\mathbf{C}_{i}^{\star} \mathbf{P}_{i+1} \mathbf{C}_{i} .
\end{aligned}
$$

In this way $J_{\min }$ can be computed a priori, without evaluating the states or the control signal. In Eq. (D18) only the first term in the right-hand side is exclusively determined by the initial condition. The second term denotes the interaction of the initial condition and forcing at the top, and the third is present only due to the external forcing.

This result refers to a single wave number only. Substituting in Eq. (36) and integrating over all wave numbers, we can compute the minimum, $E_{\min }$ that can be achieved due to the full spectrum of free-stream turbulence. 


\section{CLOSED-LOOP CONTROL OF BOUNDARY LAYER STREAKS ...}

[1] S. Bagheri, Computational hydrodynamic stability and flow control based on spectral analysis of linear operators, Arch. Comput. Methods Eng. 19, 341 (2012).

[2] S. Bagheri and D. Henningson, Transition delay using control theory, Philos. Trans. R. Soc. London A 369, 1365 (2011).

[3] S. Bagheri, D. Henningson, J. Hoepffner, and P. Schmid, Input-output analysis and control design applied to a linear model of spatialy developing flows, Appl. Mech. Rev. 62, 020803 (2009).

[4] J. Kim and T. R. Bewley, A linear systems approach to flow control, Annu. Rev. Fluid Mech. 39, 383 (2007).

[5] T. T. Medjo, R. Temam, and M. Ziane, Optimal and robust control of fluid flows: some theoretical and computational aspects, Appl. Mech. Rev. 61, 010802 (2008).

[6] L. Baramov, O. R. Tutty, and E. Rogers, $H_{\infty}$ control of non-periodic two-dimensional channel flow, IEEE Trans. Control Sys. Tech. 12, 111 (2002).

[7] T. R. Bewley and S. Liu, Optimal and robust control and estimation of linear paths to transition, J. Fluid Mech. 365, 305 (1998).

[8] J. McKernan, G. Papadakis, and J. F. Whidborne, Linear and non-linear simulations of feedback control in plane Poiseuille flow, Int. J. Num. Mech. Fluids 59, 907 (2009).

[9] B. Bamieh, F. Paganini, and M. A. Dahleh, Distributed control of spatially invariant systems, IEEE Trans. Automatic Control 47, 1091 (2002).

[10] M. Högberg and D. S. Henningson, Linear optimal control applied to instabilities in spatially developing boundary layers, J. Fluid Mech. 470, 151 (2002).

[11] M. Chevalier, J. Hoepffner, E. Akervik, and D. S. Henningson, Linear feedback control and estimation applied to instabilities in spatially developing boundary layer, J. Fluid Mech. 588, 163 (2007).

[12] P. Cathalifaud and P. Luchini, Algebraic growth in boundary layers: optimal control by blowing and suction at the wall, Eur. J. Mech. B-Fluids 19, 469 (2000).

[13] P. Cathalifaud and T. R. Bewley, A noncausal framework for model-based feedback control of spatially developing perturbations in boundary-layer flow systems. Part I: Formulation, Sys. Control Lett. 51, 1 (2004).

[14] P. Cathalifaud and T. R. Bewley, A noncausal framework for model-based feedback control of spatially developing perturbations in boundary-layer flow systems. Part II: Numerical simulations using state feedback, Syst. Control Lett. 51, 15 (2004).

[15] A. Bryson and Y. Ho, Applied Optimal Control (Taylor and Francis, Philadelphia, 1975).

[16] R. F. Stengel, Optimal Control and Estimation (Dover, New York, 1994).

[17] B. Moore, Principal component analysis in linear systems: Controllability, observability and model reduction, IEEE Trans. Autom. Control 26, 17 (1981).

[18] C. Rowley, Model reduction for fluids using balanced proper orthogonal decomposition, Int. J. Bifur. Chaos 15, 997 (2005).

[19] S. Bagheri, L. Brandt, and D. Henningson, Input-output analysis, model reduction and control of the flat plate boundary layer, J. Fluid Mech. 620, 263 (2009).

[20] O. Semerano, S. Bagheri, L. Brandt, and D. S. Henningson, Feedback control of three-dimensional optimal disturbances using reduced-order models, J. Fluid Mech. 677, 63 (2011).

[21] J. Pralits and P. Luchini, Riccati-less optimal control of bluff-body wakes, in Proc. 7th IUTAM Symposium on Laminar-Turbulent Transition Stockholm, Sweden, 2009, edited by P. Schlatter and D. Henningson (Springer, Dordrecht, 2010), pp. 325-330.

[22] O. Semerano, J. Pralits, C. Rowley, and D. Henningson, Riccati-less approach for optimal control and estimation: an application to two dimensional boundary layers, J. Fluid Mech. 731, 394 (2013).

[23] S. J. Leib, D. W. Wundrow, and M. E. Goldstein, Effect of free-stream turbulence and other vortical disturbances on laminar boundary layer, J. Fluid Mech. 380, 169 (1999).

[24] P. Davidson, Turbulence: An Introduction for Scientists and Engineers (Oxford University Press, Oxford, 2004).

[25] M. Goldstein, Response of the pre-transitional laminar boundary layer to free-stream turbulence-Otto Laporte Lecture, Bull. Am. Phys. Soc. 42, 2150 (1997). 
[26] M. E. Goldstein, Unsteady vortical and entropic distortions of potential flows around arbitrary obstacles, J. Fluid Mech. 89, 433 (1978).

[27] M. E. Goldstein, The evolution of Tollmien-Schlichting waves near a leading edge, J. Fluid Mech. 127, 59 (1983).

[28] H. Schlichting, Boundary Layer Theory (McGraw-Hill, New York, 1985).

[29] A. N. Gulyaev, V. E. Kozlov, V. R. Kuznetson, B. I. Mineev, and A. N. Sekundov, Interaction of a laminar boundary layer with external turbulence, Fluid Dyn. 24, 700 (1989).

[30] P. Ricco, The pre-transitional Klebanoff modes and other boundary layer disturbances induced by small wavelength free-stream vorticity, J. Fluid Mech. 638, 267 (2009).

[31] D. Bender and A. Laub, The linear-quadratic optimal regulator for descriptor systems, IEEE Trans. Automatic Control 32, 672 (1987).

[32] J. Hunt, A theory of turbulent flow round two dimensional bluff bodies, J. Fluid Mech. 61, 625 (1973).

[33] P. Ricco and $\mathrm{X}$. Wu, Response of a compressible laminar boundary layer to free-stream vortical disturbances, J. Fluid Mech. 587, 97 (2007).

[34] M. Green and D. Limebeer, Linear Robust Control (Prentice Hall, Englewood Cliffs, NJ, 1995).

[35] M. Högberg, T. R. Bewley, and D. S. Henningson, Linear feedback control and estimation of transition in plane channel flow, J. Fluid Mech. 481, 149 (2003).

[36] F. L. Lewis, D. L. Vrabie, and V. L. Syrmos, Optimal Control, 3rd ed. (John Wiley and Sons, New York, 2012).

[37] P. Luchini and A. Bottaro, Adjoint equations in stability analysis, Annu. Rev. Fluid Mech. 46, 493 (2014).

[38] P. Schmid and D. Henningson, Stability and Transition in Shear Flows (Springer, New York, 2001).

[39] P. Ricco, J. Luo, and X. Wu, Evolution and instability of unsteady nonlinear streaks generated by freestream vortical disturbances, J. Fluid Mech. 677, 1 (2011).

[40] D. Wundrow and M. Goldstein, Effect on a laminar boundary layer of small-amplitude streamwise vorticity in the upstream flow, J. Fluid Mech. 426, 229 (2001).

[41] M. Choudhari, Boundary layer receptivity to three-dimensional unsteady vortical disturbances in the free stream, AIAA Paper 96-0181, 1996.

[42] X. Wu and M. Choudhari, Linear and non-linear instabilities of a Blasius boundary layer perturbed by streamwise vortices. Part 2. Intermittent instability induced by long-wavelength Klebanoff modes, J. Fluid Mech. 483, 249 (2003).

[43] X. Wu and J. Luo, Linear and non-linear instabilities of a Blasius boundary layer perturbed by streamwise vortices. Part 1. Steady streaks, J. Fluid Mech. 483, 225 (2003).

[44] B. Farrell and P. Ioannou, Stochastic forcing of the linearized Navier-Stokes equations, Phys. Fluids A 5, 2600 (1993).

[45] M. Jovanovic and B. Bamieh, Componentwise energy amplification in channel flows, J. Fluid Mech. 534, 145 (2005).

[46] J. P. Boyd, Chebyshev and Fourier Spectral Methods, 2nd ed. (Dover, New York, 2001).

[47] C. Canuto, M. Hussaini, A. Quarteroni, and T. Zhang, Spectral Methods: Evolution to Complex Geometries and Applications to Fluid Dynamics (Springer, New York, 2007).

[48] P. Moin and J. Kim, On the numerical solution of time-dependent viscous incompressible fluid flows involving solid boundaries, J. Comp. Phys. 35, 381 (1980). 